\title{
COMPUTATIONAL PROBLEMS IN METRIC FIXED POINT THEORY AND THEIR WEIHRAUCH DEGREES*
}

\author{
EIKE NEUMANN
}

Technische Universität Darmstadt, Germany

e-mail address: eike.neumann@stud.tu-darmstadt.de

\begin{abstract}
We study the computational difficulty of the problem of finding fixed points of nonexpansive mappings in uniformly convex Banach spaces. We show that the fixed point sets of computable nonexpansive self-maps of a nonempty, computably weakly closed, convex and bounded subset of a computable real Hilbert space are precisely the nonempty, co-r.e. weakly closed, convex subsets of the domain. A uniform version of this result allows us to determine the Weihrauch degree of the Browder-Göhde-Kirk theorem in computable real Hilbert space: it is equivalent to a closed choice principle, which receives as input a closed, convex and bounded set via negative information in the weak topology and outputs a point in the set, represented in the strong topology. While in finite dimensional uniformly convex Banach spaces, computable nonexpansive mappings always have computable fixed points, on the unit ball in infinite-dimensional separable Hilbert space the Browder-GöhdeKirk theorem becomes Weihrauch-equivalent to the limit operator, and on the Hilbert cube it is equivalent to Weak König's Lemma. In particular, computable nonexpansive mappings may not have any computable fixed points in infinite dimension. We also study the computational difficulty of the problem of finding rates of convergence for a large class of fixed point iterations, which generalise both Halpern- and Mann-iterations, and prove that the problem of finding rates of convergence already on the unit interval is equivalent to the limit operator.
\end{abstract}

\section{INTRODUCTION}

Metric fixed point theory is the study of fixed point properties of mappings that arise from the geometric structure of the underlying space or the geometric properties of the mappings themselves. An important classical framework for metric fixed point theory is the study of nonexpansive mappings in uniformly convex Banach spaces. A Banach space $E$ is called

2012 ACM CCS: [Mathematics of computing]: Mathematical analysis; Continuous mathematics; [Theory of computation]: Logic - Constructive mathematics.

Key words and phrases: computable analysis, functional analysis, nonexpansive mappings, fixed point theory, Weihrauch degrees.

* This paper is essentially a condensed version of the author's master's thesis [61, written under the supervision of Ulrich Kohlenbach at Technische Universität Darmstadt.

The author was partly supported by the German Research Foundation (DFG) with Project Zi 1009/4-1 and by the Royal Society International Exchange Grant IE111233. 
strictly convex, if for all $x, y \in E$ with $x \neq y$ and $\|x\|=\|y\|=1$, we have $\left\|\frac{x+y}{2}\right\|<1$. It is called uniformly convex, if

$$
\forall \varepsilon \in(0,2] . \exists \delta \in(0,1] . \forall x, y \in B_{E} \cdot\left(\|x-y\| \geq \varepsilon \rightarrow\left\|\frac{x+y}{2}\right\| \leq 1-\delta\right) .
$$

Here, $B_{E}$ denotes the closed unit ball of $E$. Clearly, every uniformly convex Banach space is strictly convex. A function $\eta_{E}:(0,2] \rightarrow(0,1]$ witnessing the existential quantifier is called a modulus of convexity for $E$. By the parallelogram law, every Hilbert space $H$ is uniformly convex with computable modulus of convexity $\eta_{H}(\varepsilon)=1-\sqrt{1-\frac{\varepsilon^{2}}{4}}$. More generally, all $L^{p}$-spaces with $1<p<\infty$ are uniformly convex with a computable modulus of uniform convexity. A mapping $f: \subseteq E \rightarrow E$ is called nonexpansive if it is Lipschitz-continuous with Lipschitz-constant one, i.e. if

$$
\|f(x)-f(y)\| \leq\|x-y\| \text { for all } x, y \in \operatorname{dom} f .
$$

We have the following existence result:

Theorem 1.1 (Browder-Göhde-Kirk). Let $E$ be a uniformly convex Banach space, let $K \subseteq E$ be nonempty, bounded, closed, and convex, and let $f: K \rightarrow K$ be nonexpansive. Then $f$ has a fixed point.

Theorem 1.1] was proved independently by Browder [24], Göhde [34, and Kirk [40] in 1965 (Kirk's version is even more general than the version stated here). Throughout this paper we denote the fixed point set of a mapping $f$ by $\operatorname{Fix}(f)$. A considerable amount of attention is dedicated to the study of so-called fixed point iterations, which start with an initial guess $x_{0}$ for a fixed point of $f$ and successively improve the guess by applying a computable operation, which yields a sequence $\left(x_{n}\right)_{n}$ of points in $K$ that is then shown to converge (weakly or strongly) to a fixed point. Many of these results are modifications of either of two classical theorems.

Theorem 1.2 (Wittmann, [81]). Let $H$ be a Hilbert space, let $K \subseteq H$ be nonempty, bounded, closed, and convex, and let $f: K \rightarrow K$ be nonexpansive. Choose a starting point $x \in K$ and an "anchor point" $y \in K$ and consider the sequence $\left(x_{n}\right)_{n}$, where $x_{0}=x$ and $x_{n+1}=$ $\frac{1}{n+2} y+\left(1-\frac{1}{n+2}\right) f\left(x_{n}\right)$. Then the sequence $\left(x_{n}\right)_{n}$ converges to the uniquely defined fixed point of $f$ which is closest to the anchor point $y$.

Theorem 1.3 (Krasnoselski, [51]). Let $E$ be a uniformly convex Banach space, let $K \subseteq E$ be nonempty, bounded, closed, and convex, and let $f: K \rightarrow K$ be nonexpansive and $f(K)$ be compact. Then for any $x \in K$ the sequence $\left(x_{n}\right)_{n}$, where $x_{0}=x$ and $x_{n+1}=\left(f\left(x_{n}\right)+x_{n}\right) / 2$, converges to a fixed point of $f$.

The iteration employed in Theorem 1.2 is a special case of a general iteration scheme, typically referred to as Halpern iteration, as it was first introduced by Halpern [35. It has the general form $x_{n+1}=\left(1-\alpha_{n}\right) y+\alpha_{n} f\left(x_{n}\right)$, where $\alpha_{n} \in(0,1)$, and the iteration can be shown to converge if certain conditions are imposed on $\left(\alpha_{n}\right)_{n}$. The iteration used in Theorem 1.3 can be similarly generalised to the scheme $x_{n+1}=\left(1-\alpha_{n}\right) x_{n}+\alpha_{n} f\left(x_{n}\right)$, and again there are certain conditions that guarantee convergence. This iteration scheme is typically called Krasnoselski-Mann iteration or simply Mann iteration. In Hilbert space, Krasnoselski's iteration converges weakly to a fixed point, even in the absence of compactness (cf. [62]). While these iterations do allow us to compute a sequence of approximations 
which is guaranteed to eventually converge to a fixed point, it is well known that the requirement of mere convergence is too weak to constitute a satisfactory notion of effective approximation, as there exist for instance computable sequences of rational numbers whose limit encodes the special halting problem (cf. [72]). It is hence important to understand the quantitative convergence behaviour of the approximation sequence. Quantitative aspects of metric fixed point theory have been very successfully studied within the programme of proof mining (the standard reference is [46], see also e.g. [43, 45, 44, 57, 47, 50, 69]), which is concerned with the extraction of hidden effective data from non-effective proofs. Most of the applications of proof mining in fixed point theory focus on the extraction of either of two types of effective data. Firstly, one considers rates of asymptotic regularity of the iteration, which in this context mean rates of convergence of the sequence $\left(\left\|f\left(x_{n}\right)-x_{n}\right\|\right)_{n}$ towards zero. These allow us to compute arbitrarily good $\varepsilon$-fixed points, i.e. points $x_{\varepsilon}$ satisfying $\left\|f\left(x_{\varepsilon}\right)-x_{\varepsilon}\right\|<\varepsilon$, up to arbitrary precision with an a-priori running time estimate. Secondly, one considers so-called rates of metastability (see also [53, 54, 75, 76]), which constitute a more refined quantitative measure of approximation quality. A function $\Phi: \mathbb{N}^{\mathbb{N}} \times \mathbb{N} \rightarrow \mathbb{N}$ is called a rate of metastability for the sequence $\left(x_{n}\right)_{n}$ if it satisfies

$$
\forall n \in \mathbb{N} . \forall g: \mathbb{N} \rightarrow \mathbb{N} . \exists k \leq \Phi(g, n) . \forall i, j \in[k ; k+g(k)]\left(\left\|x_{i}-x_{j}\right\|<2^{-n}\right) .
$$

Note that (1.1) is classically (but not constructively) equivalent to the statement that $\left(x_{n}\right)_{n}$ is a Cauchy sequence, so that metastability can be viewed as a finitary version of convergence. Also note that in the case of Krasnoselski's iteration, asymptotic regularity is the special case of metastability where $g(k)=1$ for all $k \in \mathbb{N}$. Of course, both types of information are strictly weaker than actual rates of convergence. In fact, effective uniform rates of convergence cannot exist, as the existence result fails to be computably realisable already in the case where $K=[0,1]$.

Theorem 1.4 ([43]). The multi-valued operator which receives as input a nonexpansive self map $f$ of the compact unit interval $[0,1]$ and returns some fixed point of $f$ is not computable.

While Theorem 1.4 already shows that there exists no algorithm for computing a rate of convergence for Krasnoselski's or Halpern's iteration uniformly in the input function and the starting point, it leaves several questions open: whether every computable nonexpansive mapping has a computable fixed point, whether there exist non-uniformly computable rates of convergence for the Mann- or Halpern-iteration for every computable nonexpansive mapping, at least for certain suitable starting points, whether fixed points are uniformly computable relative to discrete advice, what the exact relation between the computational content of the three theorems is, and how their computational content relates to the computational content of other mathematical theorems, such as Brouwer's fixed point theorem.

In this paper, we study the computational content of Theorems 1.1, 1.3, and 1.2, as well as related computational problems in terms of Weihrauch degrees, which have been proposed by Gherardi and Brattka [17] as a framework for classifying mathematical theorems according to their computational content. Many classical mathematical theorems have been classified over the recent years. Recently, Brattka, Le Roux and Pauly [19] have shown that Brouwer's fixed point theorem in dimension $n$ is equivalent to the closed choice principle on the closed unit ball in $\mathbb{R}^{n}$ restricted to connected sets, and that it is equivalent to Weak König's Lemma from dimension three upwards. Their work is based on a characterisation of the fixed point sets of computable self-maps of the unit ball in $\mathbb{R}^{n}$, due to Miller [59]. We 
provide a similar characterisation for the fixed point sets of computable nonexpansive selfmaps of nonempty, convex, closed, and bounded subsets of computable Hilbert space, which we can use to determine the Weihrauch degree of the Browder-Göhde-Kirk theorem. This will in particular allow us to compare the computational content of the Browder-GöhdeKirk theorem and the problem of finding rates of convergence for fixed point iterations to Brouwer's classic result.

\section{Preliminaries}

Here we review some basic notions from computable and functional analysis and the theory of Weihrauch reducibility. Most of the results in this section are more or less folklore, and none of them are original, except maybe Proposition 2.15. Standard references in computable analysis are [66] and [79]. A more general treatment of the theory of computable metric spaces can be found in [15] and [20]. The results in functional analysis reviewed here can for instance be found in [58] or [80]. We will closely follow the approach to computable analysis taken by Matthias Schröder [70], and more recently by Arno Pauly [64], particularly concerning the canonical constructions of hyperspaces. Also, we adopt most of the notation and terminology from [64], which differs from standard terminology at certain points (see Caveat 2.2).

A numbering of a nonempty countable set $S$ is a surjective partial mapping $\nu: \subseteq \mathbb{N} \rightarrow S$. A representation of a nonempty set $X$ is a surjective partial mapping $\delta: \subseteq \mathbb{N}^{\mathbb{N}} \rightarrow X$. If $\delta$ is a representation of $X$, we call the tuple $(X, \delta)$ a represented space. If the underlying representation is clear from context, we will often simply write $X$ for $(X, \delta)$ and by convention denote the underlying representation $\delta$ of $X$ by $\delta_{X}$. If $\delta$ and $\varepsilon$ are representations of the same set $X$, we denote continuous reduction by $\delta \leq_{t} \varepsilon$ and computable reduction by $\delta \leq \varepsilon$. A representation $\delta: \subseteq \mathbb{N}^{\mathbb{N}} \rightarrow X$ is admissible if it is continuous and maximal with respect to continuous reduction. A represented topological space 1 is a tuple $(X, \delta)$, where $X$ is a topological space and $\delta$ is an admissible representation for $X$. If the representation is clear from the context, we will simply write $X$ for $(X, \delta)$ and by convention denote the underlying admissible representation $\delta$ by $\delta_{X}$. We say that a partial mapping $F: \subseteq \mathbb{N}^{\mathbb{N}} \rightarrow \mathbb{N}^{\mathbb{N}}$ is a realiser for a partial multi-valued mapping (or "multimapping") $f: \subseteq \bar{X} \rightrightarrows Y$ between represented spaces and write $F \vdash f$ if $\delta_{Y}(F(p)) \in f(x)$, whenever $\delta_{X}(p)=x$. We call $f$ computable if it has a computable realiser, and realiser-continuous if it has a continuous realiser. If we want to emphasise the underlying representations, we will write that $f$ is $\left(\delta_{X}, \delta_{Y}\right)$-computable or $\left(\delta_{X}, \delta_{Y}\right)$-continuous respectively. We denote by $\rho$ the standard representation of real numbers. If $\delta: \subseteq \mathbb{N}^{\mathbb{N}} \rightarrow X$ and $\varepsilon: \subseteq \mathbb{N}^{\mathbb{N}} \rightarrow Y$ are representations (or numberings), we denote by $[\delta \rightarrow \varepsilon]$ the canonical representation of the space $[X \rightarrow Y]$ of functions with continuous realiser. If $X$ and $Y$ are represented topological spaces, then $[X \rightarrow Y]$ coincides with the space $\mathscr{C}_{\text {seq }}(X, Y)$ of sequentially continuous functions from $X$ to $Y$. If $X$ is first-countable, $[X \rightarrow Y]$ furthermore coincides with the space $\mathscr{C}(X, Y)$ of continuous functions from $X$ to $Y$. Moreover, we denote by $\delta \times \varepsilon$ the canonical representation of the product space $X \times Y$, by $\delta^{\omega}$ the canonical representation of the space $X^{\mathbb{N}}$ and by $\delta^{*}$ the canonical representation of $X^{*}=\bigcup_{n \in \mathbb{N}} X^{n}$. If $\delta$ and $\varepsilon$ are representations of the

\footnotetext{
${ }^{1}$ Like Schröder, and as opposed to Pauly, we will mostly work with a specific topology for $X$ in mind. To emphasize this, we call the spaces of interest represented topological spaces, rather than "represented spaces". In general, the topology on $X$ will not be the final topology of its representation, and topological continuity may differ from realiser-continuity if the topology of $X$ is not sequential.
} 
same space $X$, we let $\delta \sqcap \varepsilon=\left.\pi_{0}(\delta \times \varepsilon)\right|^{\Delta(X)}$, where $\Delta(X)=\left\{(x, y) \in X^{2} \mid x=y\right\}$ and $\pi_{0}$ is the projection onto the first coordinate. If $K \subset X$ is a subset of $X$, we sometimes write $\delta_{K}$ for $\left.\delta_{X}\right|^{K}$.

As already mentioned, the following constructions are essentially due to [70] and [64]. For any represented space $X$, the canonical function-space construction gives rise to canonical representations of the hyperspaces of "open" and "closed" subsets of $X$, by postulating that openness corresponds to semi-decidability. Let $\mathbb{S}=\{0,1\}$ denote Sierpiński space with topology $\{\emptyset,\{0,1\},\{1\}\}$ and representation

$$
\sigma(p)=0: \Leftrightarrow p=0 .
$$

The characteristic function $\chi_{U}: X \rightarrow\{0,1\}$ of a set $U$ is defined as $\chi_{U}(x)=1: \Leftrightarrow x \in U$.

Definition 2.1. Let $X$ be a represented space. We call a set $U \subseteq X$ open, if its characteristic function $\chi_{U}: X \rightarrow \mathbb{S}$ is realiser-continuous, i.e. $\chi_{U} \in[X \rightarrow \mathbb{S}]$. A $\theta^{X}$-name of an open set $U \subseteq X$ is a $\left[\delta_{X} \rightarrow \sigma\right]$-name of its characteristic function $\chi_{U}$. The set of all open subsets of $X$ with representation $\theta^{X}$ defines the represented space $\mathscr{O}(X)$. Dually, we define the represented space $\mathscr{A}(X)$ of closed subsets of $X$ by identifying a closed set $A \subseteq X$ with its complement in $\mathscr{O}(X)$ and call the underlying representation $\psi^{X}$.

We will often just write $\psi$ for $\psi^{X}$ if the underlying space is clear from context. We call the computable points of $\mathscr{O}(X)$ semi-decidable and the computable points in $\mathscr{A}(X)$ co-semi-decidable. Note that, just like the notion of realiser continuity may differ from topological continuity, the notions of closedness and openness for subsets of represented spaces are a-priori different from the notions of topological openness and closedness. If $X$ is an admissibly represented topological space, then the set $\mathscr{O}(X)$ coincides with the set of all sequentially open subsets of $X$ and $\mathscr{A}(X)$ coincides with the set of all sequentially closed subsets of $X$. If in addition $X$ is second-countable then $\mathscr{O}(X)$ is the set of open subsets of $X$ and $\mathscr{A}(X)$ is the set of all closed subsets of $X$.

Caveat 2.2. Note that the terminology introduced here, which is mainly due to [64], is different from the usual terminology used in computable analysis, which is for instance used in Weihrauch's book [79]. In [79], the space $\mathscr{A}(X)$ is denoted by $\mathscr{A}_{>}(X)$ and its computable elements are called co-r.e. closed, rather than co-semi-decidable. Although we have introduced our $\mathscr{A}(X)$ as "the space of closed subsets" of $X$, we deliberately refrain from referring to its computable points as "computably closed", so as to avoid confusion with topological closedness on one hand, and with Weihrauch's terminology on the other. The symbol $\mathscr{A}(X)$ is used in [79] to denote the space of closed and overt subsets of $X$, to be introduced below. Also note that in the abstract we used Weihrauch's terminology.

The space $\mathscr{A}(X)$ can be thought of as "the space of closed sets encoded via negative information". The following definition provides in a certain sense a notion of "closed sets encoded via positive information".

Definition 2.3. Let $X$ be a represented space. We define the represented space $\mathscr{V}(X)$ of overt closed subsets of $X$ to be the represented space of closed subsets of $X$, where a closed set $A \subseteq X$ is represented by a $\left[\theta^{X} \rightarrow \mathbb{S}\right]$-name of the function

$$
\text { intersects? }_{A}: \mathscr{O}(X) \rightarrow \mathbb{S}, U \mapsto \begin{cases}1 & \text { if } U \cap A \neq \emptyset, \\ 0 & \text { otherwise. }\end{cases}
$$


We denote the standard representation of $\mathscr{V}(X)$ by $v^{X}$ or simply $v$ and call the computable points of $\mathscr{V}(X)$ computably overt. Computably overt closed sets are those, for which intersection with an open set can be effectively verified. The space $\mathscr{V}(X)$ hence corresponds to the space $\mathscr{A}_{<}(X)$ in [79], and hence is sometimes called the "space of closed sets, represented with positive information". In 64 it is argued that from an intrinsic perspective, the word "closed" is rather misleading, because the closure properties of the space $\mathscr{V}(X)$ differ significantly from the closure properties of closed sets (e.g. union is computable but intersection is not, the image of a computably overt set under a computable function is computably overt, but the preimage is not), and we agree with this position. Overtness is related to effective separability, which yields a convenient criterion for computable overtness (see e.g. [20, Theorem $3.8(1)]$ ).

Proposition 2.4. Let $X$ be a separable represented topological space. Define a representation $\delta_{\text {enum }}$ of the set of nonempty closed subsets of $X$ as follows:

$$
\delta_{\text {enum }}(p)=A: \Leftrightarrow \delta_{X}^{\omega}(p) \text { is dense in } A .
$$

Then $\delta_{\text {enum }} \leq\left. v\right|^{\mathscr{V}(X) \backslash\{\emptyset\}}$.

Proof. Suppose we are given a dense sequence $\left(x_{n}\right)_{n}$ in a closed set $A$, and an open set $U \in \mathscr{O}(X)$. In order to verify if $A \cap U$, check if there exists $n \in \mathbb{N}$ such that $x_{n} \in U$. This proves the claim.

Our representation $\delta_{\text {enum }}$ is called $\delta_{\text {range }}$ in [15] and [20]. Next we define the canonical representation of the hyperspace of compact subsets of a Hausdorff represented topological space $X$. In a countably based $T_{1}$ space, a compact set can be represented as a list of all its finite open covers by basic neighbourhoods. It is easy to see that this representation is characterised by the property that containment in an open set is semi-decidable. This can be used to generalise the definition to arbitrary represented topological spaces, and in fact to arbitrary represented spaces. For the sake of simplicity we restrict ourselves to the case of Hausdorff represented topological spaces.

Definition 2.5. Let $X$ be a Hausdorff represented topological space. The represented space $\mathscr{K}(X)$ of compact subsets of $X$ is the set of all compact subsets of $X$, where a compact set $K \in \mathscr{K}(X)$ is represented as a $\left[\theta^{X} \rightarrow \sigma\right]$-name of the function contained $?_{K}: \mathscr{O}(X) \rightarrow \mathbb{S}$,

$$
\text { contained }{ }_{K}(U)=1 \Leftrightarrow K \subseteq U .
$$

We denote the canonical representation of $\mathscr{K}(X)$ by $\kappa$ and call the computable points of $\mathscr{K}(X)$ computably compact. Note that, like $\mathscr{A}(X)$, our space $\mathscr{K}(X)$ only encodes "negative" information on compact sets. Weihrauch [79] hence uses the notation " $\kappa_{>}$" for our $\kappa$. Similarly as in the case of $\mathscr{A}(X)$, computable points in our $\mathscr{K}(X)$ are called "co-r.e. compact" by some authors. Definition 2.5 can be generalised to arbitrary represented spaces, essentially by using the same approach as in Definition 2.1, and calling a subset $K$ of a represented space $X$ compact if the function contained ${ }_{K}$ is an element of $[\mathscr{O}(X) \rightarrow \mathbb{S}]$. In general this will only yield a representation of the space of saturated compact sets (cf. [64]), or a multi-valued representation of the space of compact sets (cf. [70]). If $X$ is a $T_{1}$ represented topological space, then the thus obtained space $\mathscr{K}(X)$ coincides with the set of all compact subsets of the sequentialisation $\operatorname{seq}(X)$ of $X$, whose open sets are the sequentially open sets of $X$. For details see [70]. By Proposition 3.3.2 (3) in [70, the notions of compactness, sequential compactness, and compactness in the sequentialisation coincide for Hausdorff represented topological spaces, so we obtain Definition 2.5 . 
It will sometimes be convenient to work with an intrinsic notion of computable compactness for represented spaces, which we introduce next.

Definition 2.6. A Hausdorff represented topological space $X$ is called computably compact, if the mapping

is computable.

$$
\text { empty?: } \mathscr{A}(X) \rightarrow \mathbb{S}, A \mapsto \begin{cases}1 & \text { if } A=\emptyset, \\ 0 & \text { otherwise. }\end{cases}
$$

Note that the terminology used in Definitions 2.6 and 2.5 is consistent in the sense that $X$ is a computably compact space if and only if $X$ is a computable point in $\mathscr{K}(X)$. The next proposition is a converse to this in some sense.

Proposition 2.7. Let $X$ be a Hausdorff represented topological space and $K \subseteq X$ be a nonempty co-semi-decidable subset, such that the represented space $\left(K,\left.\delta_{X}\right|^{K}\right)$ is computably compact. Then $K$ is a computably compact subset of $X$, i.e. a computable point in $\mathscr{K}(X)$.

Proof. It follows immediately from the definition of $\mathscr{O}(X)$ that the mapping

$$
\cap_{K}: \mathscr{O}(X) \rightarrow \mathscr{O}(K), U \mapsto U \cap K
$$

is computable. Now, $U \supseteq K$ if and only if $K \backslash(U \cap K)=\emptyset$. It again follows from the definition, that the mapping

$$
\mathscr{O}(K) \rightarrow \mathscr{A}(K), U \mapsto K \backslash U
$$

is computable. Since $K$ is a computably compact represented space, the mapping

$$
\mathscr{A}(K) \rightarrow \mathbb{S}, A \mapsto \begin{cases}1 & \text { if } A=\emptyset, \\ 0 & \text { if } A \neq \emptyset\end{cases}
$$

is computable. It follows that the set of open subsets of $X$ containing $K$ is computably open, i.e. $K$ is computably compact.

A closed subset of a compact space is compact, and in a Hausdorff space, every compact set is closed. We have an effective counterpart of this in the theory of represented spaces. A represented space $X$ is called effectively Hausdorff if the mapping $X \rightarrow \mathscr{A}(X), x \mapsto\{x\}$ is computable.

Proposition 2.8. Let $X$ be a Hausdorff computably compact represented topological space.

(i) The mapping id: $\mathscr{A}(X) \rightarrow \mathscr{K}(X)$ is well-defined and computable.

(ii) If $X$ is effectively Hausdorff, then the mapping id: $\mathscr{K}(X) \rightarrow \mathscr{A}(X)$ is well-defined and computable.

Proof.

(i) We are given a closed set $A \in \mathscr{A}(X)$ which we want to compute as a compact set $A \in \mathscr{K}(X)$. Given an open set $U \in \mathscr{O}(X)$ we want to verify if $U \supseteq A$. In order to do so, check if $U \cup A^{C}$ covers $X$, using that $X$ is computably compact.

(ii) We are given a compact set $K \in \mathscr{K}(X)$ which we want to compute as a closed set $K \in \mathscr{A}(X)$. Given a point $x \in X$ we want to verify if $x \notin K$. In order to do so, compute $\{x\} \in \mathscr{A}(X)$, using that $X$ is effectively Hausdorff, and verify if $\{x\}^{C} \supseteq K$, using the compactness information on $K$. 
It is easy to see that the computability and well-definedness of the mapping

$$
\text { id }: \mathscr{A}(X) \rightarrow \mathscr{K}(X)
$$

characterises computably compact represented spaces (cf. also [64]).

Theorem 2.9. Let $K$ be a computably compact represented topological space, containing a computable dense sequence. Then the mapping

$$
\max :[K \rightarrow \mathbb{R}] \rightarrow \mathbb{R}, f \mapsto \max \{f(x) \mid x \in K\}
$$

is well-defined and computable.

Proof. Since $K$ is adequately represented, we have $[K \rightarrow \mathbb{R}]=\mathscr{C}_{\text {seq }}(K, \mathbb{R})$, and $K$ is sequentially compact thanks to Proposition 3.3.2 (3) in [70]. It follows that for any $f \in[K \rightarrow \mathbb{R}]$, the set $f(K)$ is sequentially compact in $\mathbb{R}$ and thus compact. This shows that max is well-defined. It remains to show that $\max (f)$ is computable relative to $f$. Let $\left(x_{n}\right)_{n}$ be a computable dense sequence in $K$. Then the sequence $\left(f\left(x_{n}\right)\right)_{n}$ is computable relative to $f$, with $\sup _{n \in \mathbb{N}} f\left(x_{n}\right)=\max (f)$. On the other hand, for every computable $b \in \mathbb{R}$, the set $U_{b}=\{x \in K \mid f(x)<b\}$ is semi-decidable relative to $f$, so that by the computable compactness of $K$, the predicate $\forall x \in K .(f(x)<b)$ is semi-decidable relative to $f$ for all $b \in \mathbb{Q}$. We can use this to construct a sequence $\left(b_{n}\right)_{n}$ of real numbers which is computable relative to $f$ and satisfies $\max (f)=\inf _{n \in \mathbb{N}} b_{n}$. Since $\max (f)$ can hence be approximated arbitrarily well "from above" as well as "from below", it is computable relative to $f$.

It follows from Theorem 2.9 that every finite dimensional uniformly convex computable Banach space $E$ has a computable modulus of uniform convexity $\eta_{E}$, since we may put

$$
\eta_{E}(\varepsilon)=\inf \left\{1-\left\|\frac{x+y}{2}\right\| \mid x, y \in B_{E},\|x-y\| \geq \varepsilon\right\},
$$

and the set $\left\{(x, y) \in B_{E} \times B_{E} \mid\|x-y\| \geq \varepsilon\right\}$ is computably compact relative to $\varepsilon$ and contains a computable, dense sequence relative to $\varepsilon$. Since the proof of Theorem 2.9 is uniform in $K$, the claim follows.

Theorem 2.10 (Kreinovich's theorem, [52]). Let $K$ be a computably compact represented topological space. Then the mapping

$$
\mathrm{UC}_{K}: \subseteq \mathscr{A}(K) \rightarrow K,\{x\} \mapsto x
$$

is computable.

Definition 2.11. A computable metric space is a triple $\left(M, d, \nu_{M}\right)$, where $(M, d)$ is a metric space and $\nu_{M}: \mathbb{N} \rightarrow A$ is a numbering of a dense subset $A \subseteq M$, such that $d: A \times A \rightarrow \mathbb{R}$ is $\left(\nu_{M} \times \nu_{M}, \rho\right)$-computable. With a computable metric space we associate the represented space $\left(M, \delta_{M}\right)$, where

$$
\delta_{M}(p)=x: \Leftrightarrow d\left(\nu_{M}(p(n)), x\right) \leq 2^{-n} \text { for all } n \in \mathbb{N} .
$$

One can show that the above defined canonical representation of a computable metric space $M$ is admissible and that $d: M \times M \rightarrow \mathbb{R}$ is computable. This canonical representation $\delta_{M}$ is also called the Cauchy representation induced by $\nu_{M}$. We will refer to the points in $\operatorname{im} \nu_{M}$ as the rational points of the represented space $M$. Note that any computable metric space is separable, and hence Hausdorff, by definition. In fact, every computable metric space is effectively Hausdorff, since the predicate $d(x, y)>0$ is a semi-decidable relative to $x$ and $y$. In any metric space $M$ we denote by $B(x, r)=\{y \in M \mid d(x, y)<r\}$ the open 
ball of radius $r$ centred at $x$, and by $\bar{B}(x, r)=\{y \in M \mid d(x, y) \leq r\}$ the closed ball of radius $r$ centred at $x$.

The following result is more or less folklore, and justifies the more abstract Definition 2.5 of computable compactness.

Proposition 2.12. A computable metric space is computably compact if and only if it is complete and computably totally bounded, i.e. if and only if there exists a function $\alpha: \mathbb{N} \rightarrow M^{*}$ such that

$$
\forall n \in \mathbb{N} . \forall x \in M . \exists k \leq \operatorname{lth}(\alpha(n)) .\left(d\left(x, \alpha(n)_{k}\right)<2^{-n}\right) .
$$

We call $\alpha(n)$ a $2^{-n}$-net in $M$.

Proof. Suppose that $M$ is computably compact. Then $M$ is compact and thus complete. Let $\left(a_{k}\right)_{k}$ be a dense computable sequence in $M$. Since $M$ is computably compact, we can verify for all $n, m \in \mathbb{N}$ if the open set $B\left(\tilde{a}_{1}, 2^{-n-1}\right) \cup \cdots \cup B\left(\tilde{a}_{m}, 2^{-n-1}\right)$ is equal to all of $M$, where $\tilde{a}_{k}$ is a rational approximation to $a_{k}$ to up error $2^{-n-1}$. In that case, $a_{1}, \ldots, a_{m}$ is a $2^{-n}$-net in $M$. On the other hand, since $\left(a_{k}\right)_{k}$ is dense in $M$ and $M$ is compact, this process has to finish after a finite number of steps for each $n \in \mathbb{N}$. It follows that $M$ is computably totally bounded.

Suppose now that $M$ is complete and computably totally bounded. Let

$$
S=\left\{B\left(c_{1}, r_{1}\right), \ldots, B\left(c_{k}, r_{k}\right)\right\}
$$

be a collection of rational balls, i.e. balls whose radii are rational numbers and whose centres are rational points in $M$. We show that we can verify if $S$ is a cover of $A$. It is a standard argument that this suffices in order to establish that $M$ is computably compact. Let $\left(\left\langle a_{1}^{n}, \ldots, a_{l(n)}^{n}\right\rangle\right)_{n \in \mathbb{N}}$ be a computable sequence of $2^{-n}$-nets in $M$. Then $S$ covers $M$ if and only if there exists $n \in \mathbb{N}$ such that for all $i \in\{1, \ldots, l(n)\}$ there exists $j \in\{1, \ldots, k\}$ such that $d\left(a_{i}^{n}, c_{j}\right)<r_{j}-2^{-n}$. This property is semi-decidable, so $M$ is computably compact.

In complete computable metric spaces, overtness is characterised by separability, in the sense that Proposition 2.4 admits a converse (cf. [20, Theorem 3.8 (2)]).

Proposition 2.13. Let $M$ be a complete computable metric space. Define the representation $\delta_{\text {enum }}$ of the set of nonempty closed subsets of $M$ as in Proposition 2.4. Then $\delta_{\text {enum }} \equiv\left(\left.v\right|^{\mathscr{V}(M) \backslash\{\emptyset\}}\right)$.

Proof. The direction $\delta_{\text {enum }} \leq v$ was already proved in Proposition 2.4. so it remains to prove $v \leq \delta_{\text {enum. }}$. Suppose we are given a closed set $A \in \mathscr{V}(M)$. We can compute an enumeration $\left(B_{m}\right)_{m}$ of all open rational balls (i.e. balls with rational centre and radius) with radius at most 1 intersecting $A$. We use this to construct a dense sequence $\left(x_{m}\right)_{m}$ in $A$. The $m^{\text {th }}$ element in the sequence is computed as follows: the first approximation $x_{m}^{(0)}$ to $x_{m}$ is the centre of $B_{m}$. Let $1 \geq \varepsilon>0$ denote the radius of $B_{m}$. We claim that we can find an open rational ball $B_{m}^{(1)}$ with radius at most $\frac{\varepsilon}{2}$ which is contained in $B_{m}$ and intersects $A$. Let $a \in B_{m} \cap A$. Then there exists rational $\frac{\varepsilon}{2}>\delta>0$ such that $d\left(a, x_{m}^{(0)}\right)<\varepsilon-\delta$. Let $\tilde{a}$ be a rational approximation of $a$ up to error $\delta / 2$. Then $d\left(\tilde{a}, x_{m}^{(0)}\right)<\varepsilon-\delta / 2$. In particular, $B(\tilde{a}, \delta / 2) \cap A \neq \emptyset$ and $B(\tilde{a}, \delta / 2) \subseteq B\left(x_{m}^{(0)}, \varepsilon\right)$. On the other hand, we can verify for a given rational $a$ and $\delta<\frac{\varepsilon}{2}$ that $d\left(a, x_{m}^{(0)}\right)<\varepsilon-\delta$ and that $B(a, \delta) \cap A \neq \emptyset$. We may hence search for such $a$ and $\delta$, and put $B_{m}^{(1)}=B(a, \delta)$ and $x_{m}^{(1)}=a$. Continuing in this manner, we obtain 
a Cauchy sequence $x_{m}^{(n)}$ with $d\left(x_{m}^{(n)}, x_{m}^{(n+k)}\right)<2^{-n}$ for all $k, n \in \mathbb{N}$. Since $M$ is complete, the sequence $x_{m}^{(n)}$ converges to some element $x_{m} \in A$ with $d\left(x_{m}^{(n)}, x_{m}\right) \leq 2^{-n}$. Applying this to all $\left(B_{m}\right)_{m}$ in parallel, we obtain a computable sequence $\left(x_{m}\right)_{m}$. It remains to show that $\left(x_{m}\right)_{m}$ is dense in $A$. Let $a \in A$, and let $\varepsilon$ be a rational number satisfying $1>\varepsilon>0$. There exists a rational point $x$ satisfying $d(a, x)<\frac{\varepsilon}{2}$. In particular, $B\left(x, \frac{\varepsilon}{2}\right) \cap A \neq \emptyset$, so $B\left(x, \frac{\varepsilon}{2}\right)=B_{k}$ for some $k \in \mathbb{N}$. It follows from the construction of $x_{k}$ that $d\left(x_{k}, a\right)<\varepsilon$.

If $M$ is a computable metric space, we have another natural notion of computability for closed sets, by identifying a closed set $A \subseteq M$ with its distance function

$$
d_{A}: M \rightarrow \mathbb{R}, d_{A}(x)=\inf \{d(x, y) \mid y \in A\} .
$$

Define represented spaces $\mathbb{R}_{<}=\left(\mathbb{R}, \rho_{<}\right)$and $\mathbb{R}_{>}=\left(\mathbb{R}, \rho_{>}\right)$via

$$
\rho_{<}(p)=x: \Leftrightarrow \sup _{n \in \mathbb{N}} \nu_{\mathbb{Q}}^{\omega}(p)(n)=x \quad \text { and } \quad \rho_{>}(p)=x: \Leftrightarrow \inf _{n \in \mathbb{N}} \nu_{\mathbb{Q}}^{\omega}(p)(n)=x .
$$

Computable elements of $\mathbb{R}_{<}$are called left-r.e. numbers, and computable elements of $\mathbb{R}_{>}$ are called right-r.e. numbers. Obviously, a number is computable if and only if it is both right- and left-r.e., whereas a classic result due to Specker [72] asserts the existence of both uncomputable left-r.e.- and uncomputable right-r.e. numbers.

Definition 2.14. Let $M$ be a computable metric space.

(i) The represented space $\mathscr{A}_{\text {dist }}(M)$ is the space of nonempty closed subsets of $M$, where a closed subset $A \subseteq M$ is represented via a $\left[\delta_{M} \rightarrow \rho\right]$-name of its distance function (2.1).

(ii) The represented space $\mathscr{A}_{\text {dist }}(M)$ is the space of nonempty closed subsets of $M$, where a closed subset $A \subseteq M$ is represented via a $\left[\delta_{M} \rightarrow \rho_{<}\right]$-name of its distance function (2.1).

(iii) The represented space $\mathscr{A}_{\text {dist }}(M)$ is the space of nonempty closed subsets of $M$, where a closed subset $A \subseteq M$ is represented via a $\left[\delta_{M} \rightarrow \rho_{>}\right]$-name of its distance function (2.1).

Computable points of $\mathscr{A}_{\text {dist }}(M)$ are called located, computable points of $\mathscr{A}_{\text {dist }}(M)$ are called lower semi-located, and computable points of $\mathscr{A}_{\text {dist }>}(M)$ are called upper semi-located. Using Proposition 2.13, it is easy to see that for any complete computable metric space $M$, the canonical representations of the spaces $\mathscr{A}_{\text {dist }}(M)$ and $\mathscr{V}(M) \backslash\{\emptyset\}$ are equivalent (see also [20. Theorem 3.7]). It is also easy to see that id: $\mathscr{A}_{\text {dist }<}(M) \rightarrow \mathscr{A}(M)$ is computable (see [20, Theorem 3.11 (1)]). In [79, Lemma 5.1.7] it is proved, that for $M=\mathbb{R}^{d}$, the canonical representations of $\mathscr{A}_{\text {dist }}(M)$ and $\mathscr{A}(M) \backslash\{\emptyset\}$ are equivalent, and the argument readily generalises to any complete computable metric space with (effectively) compact closed balls (see [20, Theorem $3.11(3)]$ ). Any such space is locally compact. Local compactness is in fact necessary for the reduction to hold:

Proposition 2.15. Let $M$ be a complete computable metric space. If the identity mapping $\mathrm{id}: \mathscr{A}(M) \backslash\{\emptyset\} \rightarrow \mathscr{A}_{\mathrm{dist}_{<}}(M)$ is computable, then $M$ is locally compact.

Proof. If $M$ is a singleton, the claim is trivial, so we may assume that $M$ consists of at least two points. Given $x \in M$ we show that we can compute $\bar{B}(x, r)$ as a compact subset of $M$, for $r$ sufficiently small. We search for a rational $y \in M$ and $r \in \mathbb{Q}_{+}$with $d(y, x)>r$. By an argument similar to Proposition 2.12, it suffices to compute for every $k \in \mathbb{N}$ a cover of $\bar{B}(x, r)$ by balls of radius $2^{-k}$, whose centres are rational points in $M$. Since $y$ is 
computable, the singleton $\{y\}$ is co-semi-decidable, so we can compute an enumeration of balls with rational centres and radii exhausting the complement of $\{y\}$ such that every ball has radius at most $2^{-k}$. We feed this enumeration into the machine computing the identity id: $\mathscr{A}(M) \backslash\{\emptyset\} \rightarrow \mathscr{A}_{\text {dist }_{<}}(M)$ and use the $\left[\delta_{M} \rightarrow \rho_{<}\right]$-name provided by the machine to compute $\mathrm{d}(x,\{y\})$ from below. After having processed finitely many balls, the machine will output the lower bound $r$ on $\mathrm{d}(x,\{y\})=d(x, y)$. This means that $\bar{B}(x, r)$ is covered by these finitely many balls, since otherwise we could force the machine computing the identity to err.

The proof of Proposition 2.15 shows that we can even compute a witness for the local compactness of $M$, namely a function $f: M \rightarrow \mathscr{K}(M)$ which maps a point $x$ to a compact closed ball containing $x$.

Definition 2.16. A (real) computable normed space is a normed real vector space $E$ together with a numbering $e: \mathbb{N} \rightarrow E$ such that $\operatorname{span}\{e(n) \mid n \in \mathbb{N}\}$ is dense in $E$ and $\left(E, d, \nu_{E}\right)$ is a computable metric space, where $d(x, y)=\|x-y\|$ and $\nu_{E}$ is a canonical notation of all (finite) $\mathbb{Q}$-linear combinations of im $e$.

A complete computable normed space is called a computable Banach space. A computable normed space which is also a Hilbert space is called a computable Hilbert space. The inner product in a computable Hilbert space is computable by the polarisation identity. A computable normed space becomes a represented space when endowed with the Cauchy representation induced by the numbering $\nu_{E}$. In this representation the vector space operations and the norm are computable functions and $0 \in E$ is a computable point of the represented space $E$. An important feature of (infinite dimensional) computable normed spaces is that without loss of generality the fundamental sequence is linearly independent (cf. [66, p. 142]).

Lemma 2.17 (Effective independence lemma). Let $(E, e)$ be an infinite dimensional computable normed space. Then there exists a computable function $f: \mathbb{N} \rightarrow \mathbb{N}$ such that $e \circ f: \mathbb{N} \rightarrow E$ has dense span in $E$ and consists of linearly independent vectors.

\section{Corollary 2.18.}

(i) Every computable real Hilbert space $H$ has a computable orthonormal basis, i.e. an orthonormal basis which is a (potentially finite) computable sequence in $H$.

(ii) Every finite dimensional computable real Hilbert space is computably isometrically isomorphic to $\mathbb{R}^{d}$ for some $d \in \mathbb{N}$. Every infinite dimensional real Hilbert space is computably isometrically isomorphic to $\ell^{2}$.

Let us now introduce some basic notions from the theory of Weihrauch degrees. We will treat this paragraph somewhat informally, as we will not need to develop the theory very far. A formal and comprehensive treatment of everything stated here can be found in [17, 13, 16], and in [19], where the Weihrauch degree of Brouwer's fixed point theorem is determined.

Definition 2.19. Let $\langle\cdot, \cdot\rangle: \mathbb{N}^{\mathbb{N}} \times \mathbb{N}^{\mathbb{N}} \rightarrow \mathbb{N}^{\mathbb{N}}$ denote some computable pairing function on Baire Space. A multimapping $g: X \rightrightarrows Y$ between represented spaces $X$ and $Y$ is said to Weihrauch reduce to $h: Z \rightrightarrows W$, in symbols $g \leq_{W} h$, if there exist computable functions $K, N: \subseteq \mathbb{N}^{\mathbb{N}} \rightarrow \mathbb{N}^{\mathbb{N}}$ such that $K\langle H N$, id $\rangle$ is a realiser of $g$, whenever $H$ is a realiser of $h$. If $f \leq_{W} g$ and $g \leq_{W} f$ we say that $g$ and $f$ are Weihrauch equivalent and write $f \equiv_{W} g$. The equivalence classes with respect to $\equiv_{W}$ are called Weihrauch degrees. 
The Weihrauch degrees together with the $\leq_{W}$-relation are known to form a bounded lattice. A very important and useful tool for studying Weihrauch degrees are so-called closed choice principles on represented spaces.

Definition 2.20. Let $X$ be a represented space.

(i) The closed choice principle on $X$ is the multimapping

$$
\mathrm{C}_{X}: \mathscr{A}(X) \backslash\{\emptyset\} \rightrightarrows X, A \mapsto A .
$$

The unique choice principle $\mathrm{UC}_{X}$ on $X$ is $\mathrm{C}_{X}$ restricted to singleton sets and the connected choice principle $\mathrm{CC}_{X}$ is $\mathrm{C}_{X}$ restricted to connected sets.

(ii) Let $X$ additionally be a closed subset of a computable Banach space $E$. We define the convex choice principle $\operatorname{ConvC}_{X}$ as the restriction of $\mathrm{C}_{X}$ to the space $\mathscr{A}^{\mathrm{co}}(X)$ of convex closed subsets of $X$.

Let us now introduce some concrete Weihrauch degrees that will be useful in our further studies. The limit operator lim: $\subseteq \mathbb{N}^{\mathbb{N}} \rightarrow \mathbb{N}^{\mathbb{N}}$ takes as input a (suitably encoded) convergent sequence $\left(p_{n}\right)_{n} \in\left(\mathbb{N}^{\mathbb{N}}\right)^{\mathbb{N}} \simeq \mathbb{N}^{\mathbb{N}}$ and outputs its limit. Weak König's Lemma, WKL, takes as input an infinite binary tree and outputs an infinite path. The intermediate value theorem, IVT, takes as input a continuous function $f:[0,1] \rightarrow \mathbb{R}$ with $f(0) \cdot f(1)<0$ and outputs some point $x \in[0,1]$ such that $f(x)=0$. Brouwer's fixed point theorem in $n$-dimensional space, $\mathrm{BFT}_{n}$, takes as input a continuous function $f:[0,1]^{n} \rightarrow[0,1]^{n}$ and outputs some fixed point of $f$. Their relation is summarised in the following

Fact 2.21.

(i) $\mathrm{WKL} \equiv{ }_{W} \mathrm{C}_{\{0,1\}^{\omega}}$.

(ii) $\mathrm{CC}_{[0,1]^{n}} \equiv_{W} \mathrm{BFT}_{n} \leq_{W}$ WKL for all $n$.

(iii) $\mathrm{IVT} \equiv_{W} \operatorname{ConvC}_{[0,1]} \equiv_{W} \mathrm{CC}_{[0,1]} \equiv_{W} \mathrm{BFT}_{1}<_{W} \mathrm{BFT}_{2} \leq_{W} \mathrm{BFT}_{3} \equiv_{W} \mathrm{WKL}$.

(iv) WKL $<_{W} \lim <_{W} \mathrm{C}_{\mathbb{N}^{N}}$.

An important property of computably compact spaces is that their closed choice principle is of low degree.

Theorem 2.22. Let $K$ be a computably compact represented topological space. Then the multimapping

$$
\mathrm{C}_{K}: \mathscr{A}(K) \backslash\{\emptyset\} \rightrightarrows K, A \mapsto A
$$

satisfies $\mathrm{C}_{K} \leq_{W}$ WKL.

Finally, we need a few observations from (computable) functional analysis. The first theorem is the so-called projection theorem, which can be found in virtually any functional analysis textbook (cf. e.g. [80, Satz V.3.2 \& Lemma V.3.3]).

\section{Theorem 2.23.}

(i) Let $E$ be a uniformly convex real Banach space and let $K \subseteq E$ be nonempty, closed, and convex. For every $x \in E$ there exists a unique $y \in K$ such that

$$
d(x, y)=d(x, K)=\inf \{d(x, z) \mid z \in K\} .
$$

We denote this element by $P_{K}(x)$. The mapping $P_{K}$ is a continuous retraction onto $K$, called the metric projection. 
(ii) Let $H$ be a real Hilbert space and $K \subseteq H$ be nonempty, closed, and convex. Then $P_{K}$ is a nonexpansive mapping, and for all $x \in H$ the element $P_{K}(x)$ is characterised by the variational inequality

$$
\left(x-P_{K}(x), y-P_{K}(x)\right) \leq 0 \text { for all } y \in K .
$$

Proof.

(i) We may assume that $x \notin K$ and $x=0$. Put $r=\inf \{\|z\||| z \in K\}>0$.

Existence: Let $\left(y_{n}\right)_{n}$ be a sequence in $K$ with $\lim _{n \rightarrow \infty}\left\|y_{n}\right\|=r$. We show that $\left(y_{n}\right)_{n}$ is a Cauchy sequence. Let $\varepsilon>0$. There exists $n \in \mathbb{N}$ such that $\left\|y_{n+k}\right\|<r+\varepsilon$ for all $k \geq 0$. We have $\frac{y_{n}+y_{n+k}}{2} \in K$ for all $k \geq 0$ and thus $\left\|\frac{y_{n}+y_{n+k}}{2}\right\| \geq r$. If $\delta \in(0,1]$ satisfies $\eta(\delta)>\frac{\varepsilon}{r+\varepsilon}$, then

$$
\left\|\frac{y_{n}+y_{n+k}}{2}\right\| \geq r>(r+\varepsilon)(1-\eta(\delta)) .
$$

Applying the contraposition of uniform convexity to $\frac{y_{n+k}}{r+\varepsilon}$ and $\frac{y_{n}}{r+\varepsilon}$ thus yields

$$
\left\|y_{n}-y_{n+k}\right\|<(r+\varepsilon) \delta \text {. }
$$

Since $\frac{\varepsilon}{r+\varepsilon} \rightarrow 0$ as $\varepsilon \rightarrow 0$, and $\eta(\delta)>0$ for all $\delta \in(0,1]$, it follows that $\left\|y_{n}-y_{n+k}\right\| \rightarrow 0$ as $n \rightarrow \infty$, i.e. the sequence $\left(y_{n}\right)_{n}$ is a Cauchy sequence. Since $K$ is closed and $E$ is complete, it converges to some element $y \in K$, which satisfies $\|y\|=\lim _{n \rightarrow \infty}\left\|y_{n}\right\|=$ $r$.

Uniqueness: Suppose that the points $y_{1}, y_{2} \in K$ with $y_{1} \neq y_{2}$ satisfy $\left\|y_{i}\right\|=r>0$. Then we have $y_{1}, y_{2} \in \bar{B}(0, r)$. Since $K$ is convex, $\frac{y_{1}+y_{2}}{2} \in K$ and since $E$ is strictly convex and $y_{1} \neq y_{2}$, we have $\left\|\frac{y_{1}+y_{2}}{2}\right\|<r$. Contradiction.

(ii) On one hand we have for all $\alpha \in[0,1]$ and $z \in K$ :

$$
\begin{aligned}
\left\|x-P_{K}(x)\right\|^{2} & \leq\left\|x-\left(\alpha z+(1-\alpha) P_{K}(x)\right)\right\|^{2} \\
& =\left(x-P_{K}(x)-\alpha\left(z-P_{K}(x)\right), x-P_{K}(x)-\alpha\left(z-P_{K}(x)\right)\right) \\
& =\left\|x-P_{K}(x)\right\|^{2}-2 \alpha\left(x-P_{K}(x), z-P_{K}(x)\right)+\alpha^{2}\left\|z-P_{K}(x)\right\|^{2}
\end{aligned}
$$

and thus $\left(x-P_{K}(x), z-P_{K}(x)\right) \leq \frac{\alpha}{2}\left\|z-P_{K}(x)\right\|^{2}$ for all $\alpha \in(0,1]$, which shows that $P_{K}$ satisfies the variational inequality. On the other hand, if $p \in K$ satisfies $(x-p, z-p) \leq 0$ for all $z \in K$, then for all $z \in K$ we have

$$
\begin{aligned}
\|x-z\|^{2} & =\|(x-p)+(p-z)\|^{2} \\
& =\|x-p\|^{2}+2(x-p, p-z)+\|p-z\|^{2} \\
& \geq\|x-p\|^{2}
\end{aligned}
$$

and thus $p=P_{K}(x)$ by Theorem 2.23. It remains to show that $P_{K}$ is nonexpansive. Let $x, y \in H$. We may assume that $x \neq y$ and $P_{K}(x) \neq P_{K}(y)$. Since $P_{K}(x), P_{K}(y) \in$ $K$, we may use the variational inequality to obtain

$$
\left(P_{K}(y)-P_{K}(x), x-P_{K}(x)\right) \leq 0
$$

and

$$
\left(P_{K}(x)-P_{K}(y), y-P_{K}(y)\right) \leq 0 .
$$

Adding both inequalities yields

$$
\left(P_{K}(y)-P_{K}(x), x-y+P_{K}(y)-P_{K}(x)\right) \leq 0 .
$$


And hence

$$
\left\|P_{K}(y)-P_{K}(x)\right\|^{2} \leq\left(P_{K}(x)-P_{K}(y), x-y\right) \leq\left\|P_{K}(x)-P_{K}(y)\right\| \cdot\|x-y\|,
$$

where the last inequality is the Cauchy-Schwarz inequality. We thus obtain

$$
\left\|P_{K}(x)-P_{K}(y)\right\| \leq\|x-y\| .
$$

The next important result is that projections onto located convex sets in uniformly convex computable Banach spaces are computable relative to a modulus of convexity. This will follow from a highly uniform proof mining result due to Kohlenbach:

Theorem 2.24 ([46, Proposition 17.4]). There exists a computable functional

$$
\Phi: \mathbb{N}^{\mathbb{N}} \times \mathbb{N} \times \mathbb{N} \rightarrow \mathbb{N}
$$

such that if $E$ is a uniformly convex normed space with modulus of uniform convexity $\eta_{E}$, $\mu$ is any functional satisfying $2^{-\mu(n)} \leq \eta_{E}\left(2^{-n}\right), K \subseteq E$ is nonempty, closed, and convex, and $x \in E$ with $d(x, K) \leq d$ then

$$
\phi(n)=\Phi(\mu, d, n)
$$

is a modulus of uniqueness for the projection onto $K$. This means that if $p, q \in K$ satisfy $\|p-x\| \leq d(x, K)+2^{-\phi(n)}$ and $\|q-x\| \leq d(x, K)+2^{-\phi(n)}$, then $\|p-q\|<2^{-n}$.

Corollary 2.25. Let $E$ be a uniformly convex computable Banach space, let $C \subseteq E$ be nonempty, convex, and computably overt. Let $\eta_{E}$ be a modulus of uniform convexity for $E$. Let $\mathscr{A}_{\text {dist }}^{\mathrm{co}}(C)$ denote the represented space of nonempty convex closed subsets of $C$, represented via their distance function. Then the mapping

$$
P: \mathscr{A}_{\text {dist }}^{\text {co }}(C) \rightarrow \mathscr{C}(C, C), K \mapsto P_{K},
$$

is computable relative to $\eta_{E}$. In fact it is computable relative to any $\mu: \mathbb{N} \rightarrow \mathbb{N}$ satisfying $2^{-\mu(n)} \leq \eta_{E}\left(2^{-n}\right)$.

Proof. Let $\mu: \mathbb{N} \rightarrow \mathbb{N}$ be such that $2^{-\mu(n)} \leq \eta_{E}\left(2^{-n}\right)$ and let $\Phi$ be the functional from Theorem 2.24, We are given a set $K \in \mathscr{A}_{\text {dist }}^{\text {co }}(C)$, a point $x \in C$ and a number $n \in \mathbb{N}$ and want to compute an approximation to $P_{K}(x)$ up to error $2^{-n}$. Since we are given the distance function to $K$, we can compute an integer upper bound $d$ to $d(x, K)$. Again using the distance function, we can compute a dense sequence in $K$. This allows us to find a point $p \in K$ with $\|p-x\| \leq d(x, K)+2^{-\Phi(\mu, d, n)}$. It follows from Theorem 2.24 that $\left\|p-P_{K}(x)\right\|<2^{-n}$.

The special case where $E$ has a computable modulus of convexity and $C=E$ yields:

Corollary 2.26 ([14]). Let $E$ be a uniformly convex computable Banach space with computable modulus of uniform convexity. Then the mapping

$$
P: \mathscr{A}_{\text {dist }}^{\text {co }}(E) \rightarrow \mathscr{C}(E, E), K \mapsto P_{K},
$$

is computable. In particular, if $K \subseteq E$ is a nonempty located and convex set, then $P_{K}$ is $\left(\delta_{E}, \delta_{E}\right)$-computable. 
The (for our purpose) most important structural feature of fixed point sets of nonexpansive mappings in strictly convex Banach spaces is that they are always convex. This is a standard exercise in functional analysis. We will prove it here anyway, to give a simple example of a proof exploiting the convexity of the underlying space.

Proposition 2.27. Let $E$ be a strictly convex Banach space, let $K \subseteq E$ be nonempty, closed, bounded, and convex. Let $f: K \rightarrow K$ be nonexpansive. Then the set $\operatorname{Fix}(f)$ is convex.

Proof. Since $f$ is continuous, it suffices to show that for each $x, y \in \operatorname{Fix}(f)$, the convex combination $\frac{x+y}{2}$ is again contained in $\operatorname{Fix}(f)$ (since then it follows that the set of dyadic convex combinations of $x$ and $y$, which is dense in the line segment joining $x$ and $y$, consists entirely of fixed points). Since $f$ is nonexpansive, we have

$$
\left\|f\left(\frac{x+y}{2}\right)-x\right\|=\left\|f\left(\frac{x+y}{2}\right)-f(x)\right\| \leq\left\|\frac{x-y}{2}\right\| .
$$

Similarly, $\left\|f\left(\frac{x+y}{2}\right)-y\right\| \leq \frac{1}{2}\|x-y\|$ and obviously the same inequality holds if we replace $f\left(\frac{x+y}{2}\right)$ by $\frac{x+y}{2}$. Now, suppose that $a, b \in E$ satisfy

$$
\|a-y\| \leq\left\|\frac{x-y}{2}\right\|,\|a-x\| \leq\left\|\frac{x-y}{2}\right\|,\|b-x\| \leq\left\|\frac{x-y}{2}\right\|,\|b-y\| \leq\left\|\frac{x-y}{2}\right\| .
$$

Then, if $a \neq b$, strict convexity yields

$$
\left\|\frac{a+b}{2}-y\right\|<\left\|\frac{x-y}{2}\right\| \text { and }\left\|\frac{a+b}{2}-x\right\|<\left\|\frac{x-y}{2}\right\|
$$

and hence

$$
\|x-y\| \leq\left\|x-\frac{a+b}{2}\right\|+\left\|y-\frac{a+b}{2}\right\|<\|x-y\|,
$$

contradiction. It follows, that $f\left(\frac{x+y}{2}\right)=\frac{x+y}{2}$.

\section{Computability of Fixed points and Rates of Convergence}

In this section we study the computability-theoretic complexity of the problems of finding fixed points of nonexpansive mappings on compact domains, and of obtaining rates of convergence of certain fixed point iterations. Let us first state some natural computational problems associated with the fixed point properties of nonexpansive mappings and determine their rough relation.

Definition 3.1. Let $E$ be a uniformly convex real Banach space, let $K \subseteq E$ be a nonempty subset of $K$, and let $\mathscr{N}(K)$ denote the set of nonexpansive self-maps of $K$. A fixed point iteration on $K$ is a mapping $I: \mathscr{N}(K) \times K \rightarrow K^{\mathbb{N}}$ such that for all $f \in \mathscr{N}(K), x \in K$, we have $\lim _{n \rightarrow \infty} I(f, x)(n) \in \operatorname{Fix}(f)$.

Definition 3.2. Let $E$ be a uniformly convex computable Banach space, let $K \subseteq E$ be nonempty, co-semi-decidable, computably overt, bounded, and convex. Let $\mathscr{N}(K)$ be the represented space of nonexpansive self-maps of $K$ with representation $\left.\left[\delta_{K} \rightarrow \delta_{K}\right]\right|^{\mathscr{N}(K)}$. Let $I: \mathscr{N}(K) \times K \rightarrow K^{\mathbb{N}}$ be a computable fixed point iteration. Consider the following computational problems: 
(i) The realiser problem for the Browder-Göhde-Kirk theorem $\mathrm{BGK}_{K}$ : Given a nonexpansive function $f: K \rightarrow K$, output a fixed point for $f$. More formally:

$$
\mathrm{BGK}_{K}: \mathscr{N}(K) \rightrightarrows K, f \mapsto \operatorname{Fix}(f) \text {. }
$$

(ii) The projection problem $\operatorname{Proj}_{K}$ : Given a nonexpansive function $f: K \rightarrow K$, and a point $x \in K$ output the metric projection of $x$ onto $\operatorname{Fix}(f)$. More formally:

$$
\operatorname{Proj}_{K}: \mathscr{N}(K) \times K \rightarrow K,(f, x) \mapsto P_{\text {Fix }(f)}(x) .
$$

(iii) The limit problem $\lim (I)$ for $I$ : given a nonexpansive function $f: K \rightarrow K$ and a starting point $x \in K$, output $\lim _{n \in \mathbb{N}} I(f, x)(n)$. More formally:

$$
\lim (I): \mathscr{N}(K) \times K \rightarrow K,(f, x) \mapsto \lim _{n \rightarrow \infty} I(f, x)(n) .
$$

(iv) The rate of convergence problem $\operatorname{Conv}_{I}$ for $I$ : given a nonexpansive function $f: K \rightarrow K$ and a starting point $x \in K$, output a rate of convergence of the sequence $(I(f, x)(n))_{n}$. More formally:

$$
\begin{aligned}
\operatorname{Conv}_{I}: & \mathscr{N}(K) \times K \rightrightarrows \mathbb{N}^{\mathbb{N}} \\
& (f, x) \mapsto\left\{\varphi \in \mathbb{N}^{\mathbb{N}} \mid \forall n \in \mathbb{N} . \forall l \geq \varphi(n) .\left\|I(f, x)(l)-\lim _{k \rightarrow \infty} I(f, x)(k)\right\|<2^{-n}\right\} .
\end{aligned}
$$

Most fixed point iterations considered in the literature are of a far more particular form than just computable mappings. This can be exploited to obtain stronger uncomputability results for particular classes of fixed point iterations. We summarise some common properties.

Definition 3.3. Let $E$ be a uniformly convex real Banach space, and let $K \subseteq E$ be nonempty, convex, closed, and bounded. Let $I: \mathscr{N}(K) \times K \rightarrow K^{\mathbb{N}}$ be a fixed point iteration.

(i) $I$ is called projective if for all $f \in \mathscr{N}(K)$ and $x \in K$, the $\operatorname{limit}_{\lim _{n \rightarrow \infty}} I(f, x)(n)$ is the unique fixed point of $f$ which is closest to $x$.

(ii) $I$ is called retractive if for all $f \in \mathscr{N}(K)$ and $x \in \operatorname{Fix}(f)$, we have $\lim _{n \rightarrow \infty} I(f, x)(n)=x$.

(iii) $I$ is called avoidant if for all $f \in \mathscr{N}(K)$ and $x \in K$, we have the implication

$$
(\exists n . f(I(f, x)(n))=I(f, x)(n)) \Rightarrow f(x)=x .
$$

(iv) $I$ is called simple if it is of the form

$$
\begin{aligned}
& I(f, x)(0)=x \\
& I(f, x)(n+1)=\sum_{k=0}^{n} \alpha_{k}^{n} I(f, x)(k)+\sum_{j, k=0}^{n} \beta_{j, k}^{n} f^{(j)}(I(f, x)(k)),
\end{aligned}
$$

with $\alpha_{k}^{n} \geq 0$ and $\beta_{k, j}^{n} \geq 0$ for all $k, n, j$.

The notion of projectiveness is well-defined thanks to Theorem 2.23 and Proposition 2.27 . Any projective fixed point iteration is clearly retractive. Note that Halpern's iteration (where by convention we always choose the anchor point to be equal to the starting point) is projective and simple and that the Krasnoselski-Mann iteration is simple, retractive, and avoidant.

\section{Proposition 3.4.}

(i) Let $I: \mathscr{N}([0,1]) \times[0,1] \rightarrow[0,1]^{\mathbb{N}}$ be a simple and retractive fixed point iteration. Then $I$ is projective when restricted to the set of all monotonically increasing functions. 
(ii) Let $E$ be a uniformly convex real Banach space, and let $K \subseteq E$ be nonempty, convex, closed, and bounded. Let $I: \mathscr{N}(K) \times K \rightarrow K^{\mathbb{N}}$ be an avoidant fixed point iteration. Then for all nonexpansive $f: K \rightarrow K$ and $x \notin \operatorname{Fix}(f), \lim _{n \rightarrow \infty} I(f, x)(n)$ is a point on the boundary of $\operatorname{Fix}(f)$.

Proof.

(i) By induction one easily verifies that if $x \leq y$, then $I(f, x)(n) \leq I(f, y)(n)$ for all monotonically increasing $f:[0,1] \rightarrow[0,1]$. It follows that

$$
\lim _{n \rightarrow \infty} I(f, x)(n) \leq \lim _{n \rightarrow \infty} I(f, y)(n) .
$$

By Proposition 2.27, the set $\operatorname{Fix}(f)$ is an interval of the form $[a, b]$, possibly with $a=b$. If $x \leq a$, then, since the iteration is retractive,

$$
\lim _{n \rightarrow \infty} I(f, x)(n) \leq \lim _{n \rightarrow \infty} I(f, a)(n)=a,
$$

so $\lim _{n \rightarrow \infty} I(f, x)(n)=a$. An analogous argument applies if $x \geq b$. It follows that the mapping $\lambda x \cdot \lim _{n \rightarrow \infty} I(f, x)(n)$ is the metric projection onto $[a, b]$, i.e. the iteration is projective.

(ii) Is trivial.

The following proposition establishes the more obvious relationships between the problems introduced in Definition 3.2.

Proposition 3.5. Let $E$ be a uniformly convex computable Banach space, let $K \subseteq E$ be nonempty, co-semi-decidable, computably overt, bounded, and convex. Then

$$
\mathrm{BGK}_{K} \leq_{W} \operatorname{Proj}_{K} \cdot
$$

If $I: \mathscr{N}(K) \times K \rightarrow K^{\mathbb{N}}$ is a computable fixed point iteration, then

$$
\operatorname{BGK}_{K} \leq_{W} \lim (I) \leq_{W} \operatorname{Conv}_{I} \leq_{W} \lim .
$$

If I is projective, then

$$
\operatorname{Proj}_{K} \leq_{W} \lim (I) .
$$

Next we prove a general upper bound on the Weihrauch degree of $\operatorname{Proj}_{K}$ (and thus of $\mathrm{BGK}_{K}$ ). We need two lemmas which constitute the main steps in Goebel's proof [32] of the Browder-Göhde-Kirk theorem (see also the proof of [80, Theorem IV.7.13]).

Lemma 3.6. Let $E$ be a uniformly convex Banach space, let $K \subseteq E$ be nonempty, convex, closed, and bounded. Then there exists a function $\varphi:(0,1) \rightarrow(0,1)$ with $\lim _{\varepsilon \rightarrow 0} \varphi(\varepsilon)=0$, such that for every nonexpansive mapping $f: K \rightarrow K$ and all $x, y \in K$ we have the implication

$$
(\|x-f(x)\|<\varepsilon \wedge\|y-f(y)\|<\varepsilon) \rightarrow\left\|\frac{x+y}{2}-f\left(\frac{x+y}{2}\right)\right\|<\varphi(\varepsilon) .
$$

Actually, $\varphi(\varepsilon)$ is given by a very simple term involving $\varepsilon$ and the modulus of uniform convexity of $E$, but we do not need this fact here.

Lemma 3.7. Let $E$ be a uniformly convex Banach space, let $K \subseteq E$ be nonempty, convex, closed, and bounded, and let $f: K \rightarrow K$ be nonexpansive. Let $A \subseteq K$ be nonempty, closed, and convex. Then $A$ intersects the fixed point set of $f$ if and only if

$$
\inf \{\|f(x)-x\| \mid x \in A\}=0 .
$$


Proof. Clearly, if $A$ intersects the fixed point set of $f$, then $\inf \{\|f(x)-x\| \mid x \in A\}=0$. On the other hand, suppose that $\inf \{\|f(x)-x\| \mid x \in A\}=0$. Let

$$
\mu(s)=\inf \{\|f(x)-x\| \mid x \in A,\|x\| \leq s\}
$$

and

$$
r=\inf \{s>0 \mid \mu(s)=0\} .
$$

Since $K$ is bounded and $\inf \{\|f(x)-x\| \mid x \in A\}=0, r$ is a well-defined real number. Let $\left(x_{n}\right)_{n}$ be a sequence in $A$ with

$$
\lim _{n \rightarrow \infty}\left\|f\left(x_{n}\right)-x_{n}\right\|=0
$$

and

$$
\lim _{n \rightarrow \infty}\left\|x_{n}\right\|=r
$$

We will show that $\left(x_{n}\right)_{n}$ is a Cauchy sequence. It then follows that $\left(x_{n}\right)_{n}$ converges to a fixed point, which proves the claim. Suppose that $\left(x_{n}\right)_{n}$ is not a Cauchy sequence. Then $r>0$ and there exists $\varepsilon>0$ and a subsequence $\left(y_{n}\right)_{n}$ of $\left(x_{n}\right)_{n}$ such that $\left\|y_{n+1}-y_{n}\right\| \geq \varepsilon$ for all sufficiently large $n$. Let $2 r \geq s>r$ be such that

$$
\left(1-\eta_{E}\left(\frac{\varepsilon}{2 r}\right)\right) s<r .
$$

For $n$ sufficiently large we have $\left\|y_{n}\right\| \leq s$, so that we have the inequalities

$$
\left\|\frac{y_{n}}{s}\right\| \leq 1,\left\|\frac{y_{n+1}}{s}\right\| \leq 1,\left\|\frac{y_{n}}{s}-\frac{y_{n+1}}{s}\right\| \geq \frac{\varepsilon}{s} .
$$

Applying uniform convexity, we obtain

$$
\left\|\frac{y_{n}+y_{n+1}}{2}\right\| \leq s\left(1-\eta_{E}\left(\frac{\varepsilon}{s}\right)\right)
$$

which yields (using that without loss of generality, $\eta_{E}$ is monotonically increasing)

$$
\left\|\frac{y_{n}+y_{n+1}}{2}\right\| \leq s\left(1-\eta_{E}\left(\frac{\varepsilon}{2 r}\right)\right)<r .
$$

Now, by Lemma 3.6 we have

$$
\lim _{n \rightarrow \infty}\left\|\frac{y_{n}+y_{n+1}}{2}-f\left(\frac{y_{n}+y_{n+1}}{2}\right)\right\|=0 .
$$

This contradicts the minimality of $r$. Hence, $\left(x_{n}\right)_{n}$ is a Cauchy sequence.

Proposition 3.8. Let $E$ be a uniformly convex computable Banach space, let $K \subseteq E$ be nonempty, co-semi-decidable, computably overt, bounded, and convex. Then we have

$$
\operatorname{Proj}_{K} \leq_{W} \lim \circ \lim .
$$

If $K$ is computably compact or $E$ is a Hilbert space, then

$$
\operatorname{Proj}_{K} \leq_{W} \lim .
$$

Proof. We are given as input a nonexpansive function $f: K \rightarrow K$ and a point $x \in K$ and want to obtain the point $p=P_{\operatorname{Fix}(f)}(x) \in K$. In the case where $E$ is a Hilbert space, we can use Halpern's iteration (1.2) to obtain a computable sequence converging to $p$ and apply lim to obtain $p$ itself.

In the case where $K$ is computably compact, we can compute $\operatorname{Fix}(f)$ as an element of $\mathscr{A}_{\text {dist }<}(K)$ (see the discussion after Definition 2.14). In particular we can compute a sequence $\left(d\left(x_{n}, \operatorname{Fix}(f)\right)_{n \in \mathbb{N}}\right.$, where $\left(x_{n}\right)_{n}$ is a computable dense sequence in $K$, as an element of $\mathbb{R}_{<}^{\mathbb{N}}$. 
Using the standard identification of $\mathbb{N}^{\mathbb{N}}$ with $\left(\mathbb{N}^{\mathbb{N}}\right)^{\mathbb{N}}$, it is easy to see that we can use a single instance of lim to obtain countably many instances of lim in parallel (cf. also e.g. [13] or [17]). Using lim, we can hence compute the sequence $\left(d\left(x_{n}, \operatorname{Fix}(f)\right)_{n \in \mathbb{N}}\right.$ as an element of $\mathbb{R}^{\mathbb{N}}$, which allows us to compute $\lambda y \cdot d(y, \operatorname{Fix}(f))$ as an element of $\mathscr{C}(K, \mathbb{R})$, since we have

for all $y, z \in K$.

$$
|d(y, \operatorname{Fix}(f))-d(z, \operatorname{Fix}(f))| \leq d(y, z)
$$

Independently, we can use the same instance of lim to obtain a modulus of uniform convexity $\eta_{E}$ for $E$ : we may put

$$
\eta_{E}(\varepsilon)=\inf \left\{1-\left\|\frac{x+y}{2}\right\| \mid x, y \in B_{E},\|x-y\| \geq \varepsilon\right\},
$$

and if $\left(x_{n}\right)_{n}$ is a computable dense sequence in $B_{E}$ (we may choose e.g. the sequence of rational points contained in the open unit ball) we have

$$
\eta_{E}(\varepsilon)=\inf \left\{1-\left\|\frac{x_{i}+x_{j}}{2}\right\| \mid i, j \in \mathbb{N},\left\|x_{i}-x_{j}\right\|>\varepsilon\right\},
$$

which is clearly limit-computable 2 in $\varepsilon$. This allows us to compute the restriction of $\eta_{E}$ to the rational numbers using countably many applications of lim. In particular we can limitcompute a function $\mu: \mathbb{N} \rightarrow \mathbb{N}$ satisfying $2^{-\mu(n)} \leq \eta_{E}\left(2^{-n}\right)$. Using the distance function of $\operatorname{Fix}(f)$ and the function $\mu$, we apply Corollary 2.25 to obtain the projection of $x$ onto $\operatorname{Fix}(f)$.

In the general case, we cannot a-priori compute $\operatorname{Fix}(f)$ as an element of $\mathscr{A}_{\text {dist }}(K)$, because of Proposition 2.15. We can however use lim to obtain $\operatorname{Fix}(f)$ as an element of $\mathscr{A}_{\text {dist }}(K)$ : since $K$ is computably overt, we can list all rational closed balls $\bar{B}(a, r)$ for which the open ball $B(a, r)$ intersects $K$. Given such a rational closed ball $\bar{B}(a, r)$ in $E$, we can compute a dense sequence in $\bar{B}(a, r) \cap K$ : choose a computable dense sequence $\left(x_{n}\right)_{n}$ in $K$ and filter out those points $x_{n}$ which satisfy $d\left(x_{n}, a\right)<r$. Using the convexity of $K$ it is easy to see that the resulting sequence is dense in $\bar{B}(a, r) \cap K$. This allows us to limit-compute

$$
\inf \{\|f(x)-x\| \mid x \in \bar{B}(a, r) \cap K\} .
$$

Again, we can do this for all suitable closed rational balls in parallel. We can then enumerate those balls $\bar{B}(a, r)$ satisfying $\inf \{\|f(x)-x\| \mid x \in \bar{B}(a, r) \cap K\}>0$, which by Lemma 3.7 is equivalent to $\bar{B}(a, r) \cap \operatorname{Fix}(f)=\emptyset$. This allows us to compute the distance function to Fix $(f)$ from below (cf. [20, Theorem $3.9(1)]$ or the proof of [79, Lemma 5.1.7]). Now we apply the limit-computable method used in the compact case above to obtain the projection. Since the Weihrauch degree of the composition of two limit-computable mappings is below $\lim \circ \lim$ (see e.g. Fact 8.2 in [18]), the result follows.

Note that if $E$ is finite dimensional, then $K$ is always computably compact, so that the stronger upper bound of Proposition 3.8 applies. We will show in Theorem 6.10 that lim is an upper bound on $\operatorname{Proj}_{K}$ in all uniformly convex and uniformly smooth computable

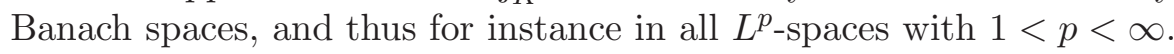

We now begin a discussion on the computability of fixed points and the Weihrauch degree of the Browder-Göhde-Kirk theorem. Proposition 2.27 yields an immediate upper bound for the Weihrauch degree of $\mathrm{BGK}_{K}$.

\footnotetext{
${ }^{2}$ We call a multimapping limit-computable if its Weihrauch degree is below lim.
} 
Proposition 3.9. Let $E$ be a uniformly convex computable Banach space. Let $K \subseteq E$ be nonempty, co-semi-decidable, computably overt, bounded, and convex. Then we have $\mathrm{BGK}_{K} \leq_{W} \operatorname{ConvC}_{K}$.

In finite dimension, this already implies that BGK is always strictly weaker than WKL. In fact it is non-uniformly computable thanks to the following result due to Le Roux and Ziegler.

Theorem 3.10 ([56]). Let $E$ be a finite dimensional computable Banach space. Let $K \subseteq E$ be nonempty, co-semi-decidable, and convex. Then $K$ contains a computable point.

Proof sketch. We may assume that $K$ is compact, since we can always intersect $K$ with a sufficiently large closed ball. We may also assume that $E$ is represented by $\rho^{d}$ for some $d \in \mathbb{N}$. We proceed by induction on $\operatorname{dim} E$. If $\operatorname{dim} E=1$, then $K$ is either a singleton and hence computable, or it is an interval and hence contains a rational point. If $\operatorname{dim} E=d$, then the projection of $K$ onto the $x$-axis is still nonempty, co-semi-decidable, and convex, and contains a computable point $x$ by induction hypothesis. Now, the intersection of $\{x\} \times \mathbb{R}^{d-1}$ with $K$ is nonempty, convex, and co-semi-decidable, of dimension strictly smaller than $d$. Again by induction hypothesis, the intersection, and in particular $K$, contains a computable point.

The above theorem even shows that $K$ has a dense subset of computable points, since the intersection of $K$ with a small rational ball is again co-semi-decidable and compact. A more uniform version, using Weihrauch degrees, has been given in [55.

Corollary 3.11. Let $E$ be a finite dimensional, strictly convex computable Banach space. Let $K \subseteq E$ be nonempty, computably overt, co-semi-decidable, bounded, and convex and let $f: K \rightarrow K$ be computable and nonexpansive. Then $f$ has a computable fixed point.

Corollary 3.11 in particular shows that the Browder-Göhde-Kirk theorem in finite dimension is strictly more effective than the (in this case more general) Brouwer fixed point theorem: a construction due to Orevkov [63] and Baigger [4] shows that there exists a computable function on the unit square in $\mathbb{R}^{2}$ without computable fixed points, while in every finite dimension some fixed points whose existence is guaranteed by the Browder-GöhdeKirk theorem are computable. Note that Corollary 3.11 really only uses the fact that $f$ is computable and its fixed point set is convex. Thus, Theorem 3.10 presents a fairly general non-uniform computability result: if a computable equation on a finite dimensional space has a convex set of solutions, then it has a computable solution. A nontrivial application is based on the following result3. A self-map $f: K \rightarrow K$ of a nonempty subset $K$ of a real Hilbert space $H$ is called pseudocontractive if it satisfies

$$
(f(x)-f(y), x-y) \leq\|x-y\|^{2}
$$

for all $x, y \in K$.

Theorem 3.12 (82]). Let $H$ be a real Hilbert space, let $K \subseteq H$ be nonempty, closed and convex, and let $f: K \rightarrow K$ be pseudocontractive. Then $\operatorname{Fix}(f)$ is closed and convex.

Corollary 3.13. Let $H$ be a finite dimensional computable Hilbert space. Let $K \subseteq H$ be nonempty, computably overt, co-semi-decidable, bounded, and convex, and let $f: K \rightarrow K$ be computable and pseudocontractive. Then $f$ has a computable fixed point.

\footnotetext{
${ }^{3}$ This application was pointed out to the author by Ulrich Kohlenbach.
} 
Corollary 3.13 is strictly more general than the Euclidean version of Corollary 3.11, as the following example, due to [28], shows.

Proposition 3.14. There exists a computable Lipschitz-continuous pseudocontractive mapping on the unit ball of Euclidean $\mathbb{R}^{2}$, which is not nonexpansive.

Proof. For $x=\left(x_{1}, x_{2}\right)$, we put $x^{\perp}=\left(-x_{2}, x_{1}\right)$. Let

$$
f(x)= \begin{cases}x+x^{\perp} & \text { if }\|x\| \leq \frac{1}{2}, \\ \frac{x}{\|x\|}-x+x^{\perp} & \text { if }\|x\| \geq \frac{1}{2} .\end{cases}
$$

It is easy to see that $f$ is computable and Lipschitz-continuous with Lipschitz constant 5 . The proof of pseudocontractiveness is somewhat technical and can be found in [28].

The function from Proposition 3.14 actually provides an example of a pseudocontractive mapping with a unique fixed point, for which the Krasnoselski-Mann iteration, which is guaranteed to converge for nonexpansive mappings, fails to converge. This is proved in [28].

In infinite dimension, there exist computable firmly nonexpansive mappings without computable fixed points, already on compact sets. A mapping $f: K \rightarrow K$ defined on a nonempty subset $K$ of a real Hilbert space $H$ is called firmly nonexpansive if it satisfies

$$
\|f(x)-f(y)\|^{2} \leq(x-y, f(x)-f(y))
$$

for all $x, y \in K$. Clearly, every firmly nonexpansive mapping is nonexpansive. It is not difficult to see that a mapping is firmly nonexpansive if and only if it is of the form $f(x)=\frac{1}{2}(x+g(x))$, where $g$ is a nonexpansive mapping. Let

$$
\mathscr{H}=\left\{x \in \ell^{2} \mid 0 \leq x(n) \leq 2^{-n} \text { for all } n \in \mathbb{N}\right\}
$$

denote the Hilbert cube in $\ell^{2}$ (represented by $\left.\delta_{\ell^{2}}\right|^{\mathscr{H}}$ ).

Theorem 3.15. There exists a computable firmly nonexpansive mapping $f: \mathscr{H} \rightarrow \mathscr{H}$ without computable fixed points.

Proof. Put $g_{n}(x)=\left(1-2^{-n}\right) x$ and $h_{n}(x)=2^{-n}+\left(1-2^{-n}\right) x$. Then $\left(g_{n}\right)_{n}$ and $\left(h_{n}\right)_{n}$ are computable sequences of nonexpansive self-maps of $[0,1]$, satisfying $\left|g_{n}(x)-x\right| \leq 2^{-n}$, $\left|h_{n}(x)-x\right| \leq 2^{-n}$ for all $x \in[0,1], \operatorname{Fix}\left(g_{n}\right)=\{0\}$, and $\operatorname{Fix}\left(h_{n}\right)=\{1\}$.

Let $A, B \subseteq \mathbb{N}$ be two disjoint, recursively enumerable, and recursively inseparable sets. Let $\alpha$ be the Gödel number of an algorithm with halting set $A$ and $\beta$ be the Gödel number of an algorithm with halting set $B$. Consider the sequence of functions $\left(f_{n}\right)_{n}$ with

$$
f_{n}(x)= \begin{cases}g_{i}(x) & \text { if } \alpha \text { halts on input } n \text { within } i \text { steps, } \\ h_{i}(x) & \text { if } \beta \text { halts on input } n \text { within } i \text { steps } \\ x & \text { if both } \alpha \text { and } \beta \text { diverge on input } n .\end{cases}
$$

Note that since $A$ and $B$ are disjoint, both $\alpha$ and $\beta$ cannot halt on the same input, so $f_{n}$ is well-defined. The sequence $\left(f_{n}\right)_{n}$ is a computable sequence: in order to compute $f_{n}(x)$ up to error $2^{-m}$, we simulate $\alpha$ and $\beta$ simultaneously on input $n$ for $m$ steps. If $\alpha$ (respectively $\beta$ ) halts within $k \leq m$ steps, we output $g_{k}(x)$ (respectively $h_{k}(x)$ ) up to error $2^{-m}$. If neither $\alpha$ nor $\beta$ halt after $m$ steps, we may output $x$ as an approximation, since $\left|g_{m+k}(x)-x\right| \leq 2^{-m-k}$ and $\left|h_{m+k}(x-x)\right| \leq 2^{-m-k}$ for all $k \geq 0$. Now, suppose there exists a computable sequence $\left(x_{n}\right)_{n}$ with $f_{n}\left(x_{n}\right)=x_{n}$. In order to arrive at a contradiction, we use $\left(x_{n}\right)_{n}$ to construct a computable set $S \subseteq \mathbb{N}$ separating $A$ and $B$. Membership for $S$ 
is decided as follows: for a given $n \in \mathbb{N}$, run the tests $x_{n}>0$ and $x_{n}<1$ simultaneously. At least one of the tests has to succeed. If the first test to succeed is $x_{n}>0$, we decide that $n \notin S$. If the first test to succeed is $x_{n}<1$, we decide that $n \in S$. Note that in the case where both $x_{n}>0$ and $x_{n}<1$, the outcome of the decision procedure may depend on the Cauchy sequence of dyadic rational numbers representing $x_{n}$. We claim that $S \supseteq A$ and $S^{C} \supseteq B$. If $n \in A$, then $\alpha$ halts on input $n$ after $i \in \mathbb{N}$ steps. So $f_{n}=g_{i}$, and thus $x_{n}=0$. The test $x_{n}>0$ will hence fail, while the test $x_{n}<1$ will succeed and thus $n \in S$. If $n \in B$, then $\beta$ halts on input $n$ after $i \in \mathbb{N}$ steps, so $f_{n}=h_{i}$ and thus $x_{n}=1$. It follows that $n \notin S$. So $S$ separates $A$ and $B$. Contradiction. Now define a nonexpansive mapping $g: \mathscr{H} \rightarrow \mathscr{H}$ via $g(x)(n)=2^{-n} f_{n}\left(2^{n} x(n)\right)$. Then $g$ is computable, for in order to compute an approximation to $g(x)$ in $\ell^{2}$ up to error $2^{-n}$, it suffices to compute the real numbers $g(x)(0), \ldots, g(x)(n+1)$ up to error $2^{-2 n-1} /(n+2)$. Any fixed point for $g$ can be used to compute a sequence of fixed points for $\left(f_{n}\right)_{n}$. In particular, $g$ has no computable fixed points. In order to obtain a firmly nonexpansive mapping $f$ we put $f=\frac{1}{2}(\mathrm{id}+g)$.

Theorem 3.15 in particular shows that fixed points of (firmly) nonexpansive mappings are not computable relative to any discrete advice. Let us now consider the computability of rates of convergence of certain fixed point iterations. While in infinite dimension, the non-uniform uncomputability of fixed points in particular implies that there exist computable mappings such that no computable fixed point iteration has a computable rate of convergence for any computable starting point, and Theorem 1.4 tells us that there is no general algorithm for obtaining rates of convergence uniformly in the input function and in the starting point, it might still be the case (at least in finite dimension) that there exists a computable fixed point iteration $I$ such that for every computable nonexpansive function $f$ there exists a computable starting point $x_{0} \notin \operatorname{Fix}(f)$ such that the sequence $I\left(f, x_{0}\right)$ has a computable rate of convergence. This could still be practically relevant, since in a given practical scenario one might be able to exploit additional information on the input function in order to choose the starting point of the iteration in such a way that the rate of convergence becomes computable. We will however see that this fails to be the case for a large class of fixed point iterations, already on the compact unit interval.

We prove a special case of our main result (Theorem 5.1) where the underlying set is the the compact unit interval $[0,1]$. Our theorem uniformly characterises the fixed point sets of computable nonexpansive self maps of $[0,1]$. We first recall an elementary fact (cf. [79]).

Proposition 3.16. Let $\mathscr{I}=\left\{(a, b) \in\left(\mathbb{R}_{<} \times \mathbb{R}_{>}\right) \mid a \leq b\right\}$. Then the mapping

$$
\mathscr{A}^{\mathrm{co}}(\mathbb{R}) \backslash\{\emptyset\} \rightarrow \mathscr{I},[a, b] \mapsto(a, b)
$$

and its inverse

$$
\mathscr{I} \rightarrow \mathscr{A}^{\mathrm{co}}(\mathbb{R}) \backslash\{\emptyset\},(a, b) \mapsto[a, b]
$$

are computable.

Theorem 3.17.

(i) The mapping

$$
\text { Fix }: \mathscr{N}([0,1]) \rightarrow \mathscr{A}^{\mathrm{co}}([0,1]) \backslash\{\emptyset\}, f \mapsto \operatorname{Fix}(f)
$$

is computable.

(ii) And so is its multivalued inverse

$$
\operatorname{Fix}^{-1}: \mathscr{A}^{\mathrm{co}}([0,1]) \backslash\{\emptyset\} \rightrightarrows \mathscr{N}([0,1]) .
$$


Proof. The first claim immediately follows from Proposition 2.27 and the well-known result that the set of zeroes of a continuous mapping $f$ is co-semi-decidable in $f$.

Let us now prove the second claim. Suppose we are given a nonempty, closed interval $[a, b] \in \mathscr{A}^{\mathrm{co}}([0,1])$. By Proposition 3.16 we can compute a monotonically increasing list $\left(a_{n}\right)_{n}$ of rational numbers converging from below to $a$, and a monotonically decreasing list $\left(b_{n}\right)_{n}$ of rational numbers converging from above to $b$. We may assume without loss of generality that $a_{n} \geq 0$ and $b_{n} \leq 1$ for all $n \in \mathbb{N}$.

From $\left(a_{n}\right)$ and $\left(b_{n}\right)$ we can compute a sequence $\left(f_{n}\right)_{n}$ of nonexpansive functions via

$$
f_{n}(x)= \begin{cases}a_{n} & \text { if } x \leq a_{n} \\ x & \text { if } a_{n} \leq x \leq b_{n} \\ b_{n} & \text { if } x \geq b_{n}\end{cases}
$$

Finally, we compute

$$
f(x)=\sum_{n \in \mathbb{N}} 2^{-n-1} f_{n}(x) .
$$

Then $f$ is nonexpansive, and maps $[0,1]$ into $[0,1]$. Let us now show that $\operatorname{Fix}(f)=[a, b]$. If $x \in[a, b]$ then $f_{n}(x)=x$ for all $n \in \mathbb{N}$, so $f(x)=\sum_{n \in \mathbb{N}} 2^{-n-1} x=x$. Suppose now without loss of generality that $x<a$. Then $f_{n}(x) \geq x$ for all $n \in \mathbb{N}$, and there exists $m \in \mathbb{N}$ such that $x<a_{m}$ and hence $f_{m}(x)>x$. It follows that

$$
f(x)=\sum_{n \in \mathbb{N}} 2^{-n-1} f_{n}(x)>x
$$

and hence $x \notin \operatorname{Fix}(f)$. An analogous argument applies if $x>b$. We may hence put $\operatorname{Fix}^{-1}([a, b])=f$.

From the proof we obtain the following non-uniform corollary, which is slightly stronger than the non-uniform version of Theorem 3.17 .

Corollary 3.18. Let $[a, b] \subseteq[0,1]$ be a co-semi-decidable interval. Then there exists a monotonically increasing, firmly nonexpansive, computable function $f:[0,1] \rightarrow[0,1]$ with $\operatorname{Fix}(f)=[a, b]$.

Proof. The algorithm we use in the proof of Theorem 3.17 to compute Fix $^{-1}$ maps any nonempty co-semi-decidable interval to a monotonically increasing, nonexpansive function $g:[0,1] \rightarrow[0,1]$ with $\operatorname{Fix}(g)=[a, b]$. In order to obtain a firmly nonexpansive function $f$, we put $f(x)=\frac{1}{2}(x+g(x))$.

Since by Proposition 3.16 any left-r.e. number can be the left endpoint of a co-semidecidable interval, we obtain the announced result together with Proposition 3.4.

Corollary 3.19. Let $I: \mathscr{N}([0,1]) \times[0,1] \rightarrow[0,1]^{\mathbb{N}}$ be an either projective, or simple and retractive, or avoidant computable fixed point iteration. Let $\varepsilon>0$. There exists a computable, firmly nonexpansive function $f:[0,1] \rightarrow[0,1]$ with $\operatorname{diam}(\operatorname{Fix}(f))<\varepsilon$ such that for no computable $x \notin \operatorname{Fix}(f)$, the sequence $I(f, x)$ has a computable rate of convergence.

Proof. Let $a \in(0,1)$ be an uncomputable left-r.e. number and $b \in(0,1)$ be an uncomputable right-r.e. number with $|a-b|<\varepsilon$. Then the closed interval $[a, b] \subseteq[0,1]$ is co-semi-decidable by Proposition 3.16, Using Corollary 3.18 we obtain a monotonically increasing firmly nonexpansive function $f:[0,1] \rightarrow[0,1]$ with $\operatorname{Fix}(f)=[a, b]$. If $x \notin \operatorname{Fix}(f)$, then by Proposition 3.4, we have $\lim _{n \rightarrow \infty} I(f, x)(n) \in\{a, b\}$. In particular, $\lim _{n \rightarrow \infty} I(f, x)(n)$ is uncomputable. 
Since $x$ is computable, the sequence $(I(f, x)(n))_{n}$ is a computable sequence of real numbers, so if it had a computable rate of convergence, its limit would be computable. This proves the claim.

Both the Halpern iteration and the Krasnoselski-Mann iteration are simple and retractive, so Corollary 3.19 applies to them. Using Weihrauch degrees, we can state our present results more uniformly.

Proposition 3.20. We have $\operatorname{Proj}_{[0,1]} \equiv_{W} \lim$. If $I: \mathscr{N}([0,1]) \times[0,1] \rightarrow[0,1]^{\mathbb{N}}$ is an either projective, or simple and retractive, or avoidant computable fixed point iteration, then $\lim (I) \equiv_{W} \operatorname{Conv}_{I} \equiv_{W} \lim$.

Proof. It is well known that the identity id: $\mathbb{R}_{<} \rightarrow \mathbb{R}$ is Weihrauch-equivalent to lim, even when restricted to the unit interval. Given $a \in \mathbb{R}_{<} \cap[0,1]$, we can compute the interval $[a, 1] \in \mathscr{A}^{\mathrm{co}}([0,1])$, and hence, by Theorem [3.17, construct a nonexpansive function $f:[0,1] \rightarrow[0,1]$ with $\operatorname{Fix}(f)=[a, 1]$. Now, $\operatorname{Proj}_{[0,1]}(f, 0)=a$, so $\lim \leq_{W} \operatorname{Proj}_{[0,1]}$. Together with Proposition 3.5 we obtain $\operatorname{Proj}_{[0,1]} \equiv_{W}$ lim. If $I$ is a projective, simple and retractive, or avoidant, computable fixed point iteration, then by Proposition 3.4 we obtain $\operatorname{Proj}_{[0,1]} \leq_{W} \lim (I)$, and thus $\lim \leq_{W} \lim (I) \leq_{W} \operatorname{Conv}_{I} \leq_{W} \lim$, i.e. $\lim (I) \equiv_{W} \operatorname{Conv}_{I} \equiv_{W} \lim$.

Proposition 3.21. $\mathrm{BGK}_{[0,1]} \equiv_{w} \operatorname{ConvC}_{[0,1]} \equiv_{W} \mathrm{IVT} \equiv_{W} \mathrm{BFT}_{1}$.

The equivalence $\mathrm{BGK}_{[0,1]} \equiv_{W} \mathrm{ConvC}_{[0,1]}$ follows immediately from Theorem 3.17, the equivalence ConvC $[0,1] \equiv_{W}$ IVT $\equiv_{W} \mathrm{BFT}_{1}$ was already stated in Fact 2.21, So far, there seems to be a significant discrepancy between the computational content of the existence result BGK and the "constructive" theorems by Wittmann and Krasnoselski. We will see in Section 5 that this discrepancy disappears on non-compact domains in infinite-dimensional Hilbert space, where the Browder-Göhde-Kirk theorem is Weihrauch equivalent to lim, and hence to Wittmann's theorem.

\section{WEAK TOPOLOGIES}

In order to be able to prove our main result in full generality, we have to introduce an admissible representation for the weak topology on a reflexive Banach space $E$. Such a representation has first been introduced by Brattka and Schröder [21]. We denote the continuous dual of a normed space $E$ by $E^{\prime}$ and define the mapping

$$
(\cdot, \cdot): E \times E^{\prime} \rightarrow \mathbb{R},\left(x, x^{\prime}\right) \mapsto x^{\prime}(x) .
$$

Definition 4.1. Let $E$ be a computable Banach space. The represented space $E_{w}^{\prime}$ is the space $E^{\prime}$, represented via the co-restriction of $\left[\delta_{E} \rightarrow \rho\right]$ to all continuous linear functionals.

Theorem $4.2([21])$. The representation $\left.\left[\delta_{E} \rightarrow \rho\right]\right|^{E^{\prime}}$ is admissible with respect to the weak* topology on $E^{\prime}$. 
Since the points of $E$, viewed as functionals on $E^{\prime}$, separate the points of $E^{\prime}$, the weak* topology on $E^{\prime}$ is Hausdorff. By Theorem 4.2, the space $E_{w}^{\prime}$ is then a Hausdorff represented topological space, so that the space $\mathscr{K}\left(E_{w}^{\prime}\right)$ is well-defined and coincides extensionally with the set of all weak* compact subsets of $E^{\prime}$. Note that this crucially relies on the separability of $E$, since since weak* sequential compactness and weak* compactness need not coincide on duals of inseparable spaces. The fact that they do coincide in the separable case also follows from the well-known fact that the weak* topology on the dual space of a separable Banach space is metrisable on the unit ball (cf. Theorem 4.4 below). Also note that the weak ${ }^{*}$ topology on $E^{\prime}$ is in general not sequential (i.e. there exist sequentially weak* closed sets which are not weak* closed). Consequently, the spaces $\mathscr{O}\left(E_{w}^{\prime}\right)$ and $\mathscr{A}\left(E_{w}^{\prime}\right)$ do not coincide with the hyperspaces of weak* open and weak* closed sets respectively, but with the hyperspaces of weak* sequentially open and weak* sequentially closed sets. If $A \in \mathscr{A}\left(E_{w}^{\prime}\right)$, we write $A_{w}$ for the represented space $\left(A,\left.\delta_{E_{w}^{\prime}}\right|^{A}\right)$ to emphasize the underlying representation.

If $E$ is a reflexive real Banach space with computable dual $E^{\prime}$, we obtain a canonical representation for $E$ with respect to the weak topology, by identifying $E$ with $E^{\prime \prime}$ and putting $E_{w}=\left(E^{\prime}\right)_{w}^{\prime}$, i.e. $E_{w}$ is the represented space $E^{\prime \prime}$ with representation $\left.\left[\delta_{E^{\prime}} \rightarrow \rho\right]\right|^{E^{\prime \prime}}$ (using that in this case the weak* topology on $E^{\prime \prime}$ coincides with the weak topology on $E$ ). Again, the space $\mathscr{K}\left(E_{w}\right)$ and the space of weakly compact subsets of $E$ coincide extensionally $\mathbb{A}^{4}$ but the caveat on $\mathscr{O}\left(E_{W}^{\prime}\right)$ and $\mathscr{A}\left(E_{w}^{\prime}\right)$ also applies to $\mathscr{O}\left(E_{w}\right)$ and $\mathscr{A}\left(E_{w}\right)$. As in the case of $E_{w}^{\prime}$, if $A \in \mathscr{A}\left(E_{w}\right)$, we write $A_{w}$ for the represented space $\left(A, \delta_{E_{w}}\right)$. We will often use the adjective "weak" when referring to elements in hyperspaces constructed from $E_{w}$. For instance, we may call the computable points of $\mathscr{A}\left(E_{w}\right)$ "weakly co-semi-decidable" and the computable points of $\mathscr{A}\left(E_{w}^{\prime}\right)$ "weak* co-semi-decidable" etc.

Remark 4.3. Note that in the definition of $E_{w}$ we only require $E^{\prime}$, but not $E$ itself, to be a computable Banach space. By definition, the mapping $(\cdot, \cdot)$ is $\left(\delta_{E_{w}} \times \delta_{E^{\prime}}, \rho\right)$-computable. If both $E$ and $E^{\prime}$ are computable Banach spaces, it is natural to require that the mapping $(\cdot, \cdot)$ be $\left(\delta_{E} \times \delta_{E^{\prime}}, \rho\right)$-computable, so that id: $E_{w} \rightarrow E$ becomes computable (see for instance Theorem 4.4 (iii), Corollary 4.5 and Propositions 4.8, 4.13 and 4.15 below). This is for instance the case for the spaces $L^{p}([0,1])$ with $1<p<\infty$, since we have $\left(L^{p}([0,1])\right)^{\prime}=$ $L^{q}([0,1])$, where $\frac{1}{p}+\frac{1}{q}=1$, and if $f \in L^{p}([0,1])$ and $g \in L^{q}([0,1])$, then $(f, g)$ is given by the effective formula

$$
(f, g)=\int_{0}^{1} f(x) g(x) \mathrm{dx} .
$$

Next, we prove some basic properties of the space $\mathscr{K}\left(E_{w}^{\prime}\right)$. We will need a few effective counterparts to classical results from functional analysis. The first is an effective version of the separable Banach-Alaoglou theorem, which was proved by Brattka [12].

Theorem 4.4 (Computable separable Banach-Alaoglou theorem, [12]). Let E be a computable Banach space. Let $B_{E_{w}^{\prime}}$ denote the unit ball in $E^{\prime}$, viewed as a subset of the represented space $E_{w}^{\prime}$ (thus bearing the weak* topology). Then

(i) $B_{E_{w}^{\prime}} \in \mathscr{K}\left(E_{w}^{\prime}\right)$.

(ii) More generally, let $K \subseteq E_{w}^{\prime}$ be a co-semi-decidable subset of $E_{w}^{\prime}$. If $K$ is bounded, then $K$ is computably weak* compact.

\footnotetext{
${ }^{4}$ Thus, Theorem 4.2 together with Proposition 3.3.2 (3) in 70 provide an interesting proof of the separable Eberlein- $\breve{S}$ mulian theorem as well as its analogue for the weak* topology in duals of separable spaces.
} 
(iii) If $E^{\prime}$ is a computable Banach space and $(\cdot, \cdot)$ is $\left(\delta_{E} \times \delta_{E^{\prime}}, \rho\right)$-computable, then $B_{E_{w}^{\prime}}$ admits the structure of a computably compact computable metric space.

Proof. It is proved in [12] that there exists a computable embedding $i: B_{E_{w}^{\prime}} \rightarrow X$ into a computably compact computable metric space $X$, such that $i\left(B_{E_{w}^{\prime}}\right)$ is computably compact as a subset of $X$ and the partial inverse $i^{-1}: i\left(B_{E_{w}^{\prime}}\right) \rightarrow B_{E_{w}^{\prime}}$ is computable. It follows that $i\left(B_{E_{w}^{\prime}}\right)$ is a computably compact represented space, and $i$ induces a computable isomorphism between $B_{E_{w}^{\prime}}$ and $i\left(B_{E_{w}^{\prime}}\right)$, so that $B_{E_{w}^{\prime}}$ is computably compact as a represented space. It follows from Proposition 2.7 that $B_{E_{w}^{\prime}}$ is a computably compact subset of $E_{w}^{\prime}$. This proves the first claim.

For the second claim, observe that the mappings

$$
\text { mult: }(0, \infty) \times \mathscr{A}\left(E_{w}^{\prime}\right) \rightarrow \mathscr{A}\left(E_{w}^{\prime}\right),(\alpha, A) \mapsto \alpha A=\{\alpha x \mid x \in A\}
$$

and

$$
\text { mult: }(0, \infty) \times \mathscr{K}\left(E_{w}^{\prime}\right) \rightarrow \mathscr{K}\left(E_{w}^{\prime}\right),(\alpha, K) \mapsto \alpha K=\{\alpha x \mid x \in K\}
$$

are computable. Proposition 2.8 (i) asserts that

$$
f: \mathscr{A}\left(B_{E_{w}^{\prime}}\right) \rightarrow \mathscr{K}\left(B_{E_{w}^{\prime}}\right), A \mapsto A
$$

is computable. Trivially, given $A \in \mathscr{A}\left(E_{w}^{\prime}\right)$, such that $A \subseteq B_{E_{w}^{\prime}}$, we can compute $A$ as a set in $\mathscr{A}\left(B_{E_{w}^{\prime}}\right)$. Given a bounded set $A \in \mathscr{A}\left(E_{w}^{\prime}\right)$ with bound $b$, we hence obtain $A$ as an element of $\mathscr{K}\left(E_{w}^{\prime}\right)$ by computing $\operatorname{mult}\left(b, f\left(\operatorname{mult}\left(\frac{1}{b}, A\right)\right)\right)$.

The third claim follows immediately from the proof of the first. We may pull back the metric $d_{X}$ on $X$ via $i$ to obtain a metric on $B_{E_{w}^{\prime}}$, i.e. put $d(x, y)=d_{X}(i(x), i(y))$ for $x, y \in B_{E_{w}^{\prime}}$. As the set of rational points in $B_{E_{w}^{\prime}}$ we may choose those rational points of the computable Banach space $E^{\prime}$ whose norm is strictly smaller than one. One now easily verifies that $B_{E_{w}^{\prime}}$ is computably compact as a computable metric space, and that the Cauchy representation on $B_{E_{w}^{\prime}}$ is computably equivalent to $\left.\left[\delta_{E} \rightarrow \rho\right]\right|^{B_{E_{w}^{\prime}}}$.

As a corollary we get an effective version of a classical result in functional analysis (cf. [80, Korollar VIII.3.13]) in the reflexive case.

Corollary 4.5. Let $E$ be a reflexive computable Banach space with computable dual $E^{\prime}$, such that the mapping $(\cdot, \cdot)$ is $\left(\delta_{E} \times \delta_{E^{\prime}}, \rho\right)$-computable. Then $E$ is computably isometrically isomorphic to a co-semi-decidable and computably overt subspace of a function space $\mathscr{C}(M)$ over a computably compact metric space $M$.

Proof. By Theorem 4.4 (iii), $M=B_{E_{w}^{\prime}}$ (with the weak* topology) is a computably compact computable metric space. We show that the mapping

$$
i: E \rightarrow \mathscr{C}(M), x \mapsto \lambda x^{\prime} \cdot\left(x, x^{\prime}\right)
$$

is a $\left(\delta_{E},\left[\delta_{B_{E_{w}^{\prime}}} \rightarrow \rho\right]\right)$-computable isometric embedding with co-semi-decidable and computably overt image. The $\left(\delta_{E},\left[\delta_{B_{E_{w}^{\prime}}} \rightarrow \rho\right]\right)$-computability is obvious, and the fact that it is an isometry follows from $\|x\|=\sup _{x^{\prime} \in B_{E^{\prime}}}\left|\left(x, x^{\prime}\right)\right|$, which in turn is an easy corollary of the Hahn-Banach theorem (cf. e.g. [80, Korollar III.1.7]). Clearly, $i(E)$ is computably overt. It is also co-semi-decidable, for if we are given a continuous function $f$ on $B_{E_{w}^{\prime}}$, we can verify if it is nonlinear. It remains to show that its inverse $i^{-1}: i(E) \rightarrow E$ is computable. Given a 
$\left[\delta_{B_{E_{w}^{\prime}}} \rightarrow \rho\right]$-name of $i(x) \in i(E)$ and a $\nu_{E}$-name of a rational point $y \in E$ we can compute a $\left[\delta_{B_{E_{w}^{\prime}}} \rightarrow \rho\right]$-name of $i(x)-i(y)$, and hence compute

$$
\max \left\{\left|\left(x-y, x^{\prime}\right)\right| \mid x^{\prime} \in B_{E_{w}^{\prime}}\right\}=\|i(x)-i(y)\|=\|x-y\|,
$$

using Theorem 2.9. For every $n \in \mathbb{N}$ we may hence search for a rational point $y_{n}$ in $E$ satisfying $\left\|y_{n}-x\right\|<2^{-n}$, which allows us to compute a $\delta_{E}$-name of $x$.

Remark 4.6. In Corollary 5 in [21], it is claimed that the representation $\delta_{E}^{w}$ of $E$ defined by

$$
\delta_{E}^{w}(p)=x: \Leftrightarrow\left[\delta_{E}^{\prime} \rightarrow \rho\right](p)=\iota(x),
$$

where $\iota: E \rightarrow E^{\prime \prime}$ is the canonical embedding and $\delta_{E}^{\prime}=\left.\left[\delta_{E} \rightarrow \rho\right]\right|^{E^{\prime}}$, is admissible for the weak topology on $E$. This contradicts the proof of our Corollary 4.5, which suggests that $\delta_{E}^{w}$ is admissible with respect to the norm topology. To convince ourselves that the claim is false, we consider the simple example of $E=\ell^{2}$. For the scope of this remark we will adopt the notation used in [21]. The representation $\delta_{\ell^{2}}^{\geq}$, where a $\delta_{\ell^{2}}^{\geq}$-name of $\left(x_{n}\right)_{n} \in \ell^{2}$ is a $\rho^{\omega}$-name of a sequence $\left(b, x_{1}, x_{2}, \ldots\right)$ with $b \geq\left\|\left(x_{n}\right)_{n}\right\|$, is equivalent to $\delta_{\ell_{w}^{2}}$ (which is denoted by $\delta_{\ell^{2}}^{\prime}$ in [21]). It follows that given a $\delta_{E}^{w}(p)$-name of $x \in \ell^{2}$ we can compute $\left(x, x^{\prime}\right)$ for any $x^{\prime} \in \ell^{2}$, provided that we know $x^{\prime}(n)$ for all $n \in \mathbb{N}$ and some bound on $\left\|x^{\prime}\right\|$. In particular we can compute $x(n)=\left(x, e_{n}\right)$ for every $n \in \mathbb{N}$. Since $\delta_{\ell^{2}} \leq \delta_{\ell_{w}^{2}}$, a $\delta_{\ell^{2}}^{w}$-name of $x$ allows us to compute a $\left[\delta_{\ell^{2}} \rightarrow \rho\right]$-name of $x$. Theorem 5.1. in [11] then asserts that we can compute some bound $b$ on $\|x\|$. It follows that we can compute $\|x\|^{2}=(x, x)$, and so $\delta_{\ell^{2}}^{w} \equiv \delta_{\ell^{2}}^{\bar{z}}$, which entails that in fact $\delta_{\ell^{2}}^{w}$ is admissible with respect to the (strictly stronger) norm topology.

The flaw in the argument seems to be the claim that for every compatible representation $\delta$ of a separable Banach space $X$, the dual representation $\delta^{\prime}=\left.\left[\delta \rightarrow \delta_{\mathbb{F}}\right]\right|^{X^{\prime}}$ is admissible with respect to the weak* topology on $X^{\prime}$, the reasoning being that for represented topological spaces $A$ and $B$, the canonical function space representation $\left[\delta_{A} \rightarrow \delta_{B}\right]$ is admissible with respect to the sequentially-compact-open topology on $[A \rightarrow B]$, and that weak*-convergence on $X^{\prime}$ coincides with compact-open-convergence on $\mathscr{C}(X, \mathbb{F})$ (cf. Theorem 4.2 and Proposition 1 in [21]). However, if $\delta$ is admissible with respect to the weak topology on $X$, then $\delta^{\prime}=[\delta \rightarrow \rho]$ is admissible with respect to the weakly-compact-open topology and not necessarily with respect to the (norm-)compact-open topology. Thus, if $X$ is a reflexive computable Banach space, and we start with the standard representation $\delta_{X}$ of $X$, which is compatible and admissible with respect to the strong topology, then $\delta_{X}^{\prime}=\left[\delta_{X} \rightarrow \rho\right]$ is compatible and admissible with respect to the compact-open topology on $X^{\prime}$, which is just the weak* topology. Applying the construction again, we see that $\left(\delta_{X}^{\prime}\right)^{\prime}=\left[\delta_{X}^{\prime} \rightarrow \rho\right]$ is compatible and admissible with respect to the weak*-compact-open topology, which in general is strictly stronger than the (norm-)compact-open-topology.

Let us now turn to some special properties of convex sets. Mazur's lemma asserts that a convex set is weakly sequentially closed if and only if it is strongly closed.

Theorem 4.7 (Mazur's lemma). Let $E$ be a Banach space and $K \subseteq E$ be convex. If $\left(x_{n}\right)_{n}$ is a sequence in $K$ which converges weakly to $x \in E$, then there exists a sequence of finite convex combinations of the $x_{n}$ 's, converging strongly to $x$.

It follows that strongly overt convex sets are weakly overt. 
Proposition 4.8. Let $E$ be a reflexive computable Banach space with computable dual $E^{\prime}$, such that the mapping $(\cdot, \cdot)$ is $\left(\delta_{E} \times \delta_{E^{\prime}}, \rho\right)$-computable. Then the identity

$$
\text { id: } \mathscr{V}^{\mathrm{co}}(E) \backslash\{\emptyset\} \rightarrow \mathscr{V}^{\mathrm{co}}\left(E_{w}\right) \backslash\{\emptyset\}
$$

where $\mathscr{V}^{\mathrm{co}}(E)$ denotes the hyperspace of convex overt closed subsets of $E$, is well-defined and computable.

Proof. The mapping id is well-defined by Theorem 4.7. Since $E$ is a computable metric space and $E_{w}$ is separable, we may use the characterisation of overtness given in Proposition 2.13 and the sufficient condition given in Proposition 2.4. If $K \in \mathscr{V}^{\mathrm{co}}(E)$, then by Proposition 2.13. we can compute a $\delta_{E}^{\omega}$-name of a norm-dense sequence $\left(x_{n}\right)_{n}$ in $K$. Since the weak topology is coarser than the norm topology, the weak sequential closure of $\left(x_{n}\right)_{n}$ contains $K$, and by Theorem 4.7, any weak limit of $\left(x_{n}\right)_{n}$ is already contained in $K$, so that $K$ is the closure of $\left(x_{n}\right)_{n}$ with respect to the sequentialisation of the weak topology. Since $(\cdot, \cdot)$ is computable, we have $\delta_{E} \leq \delta_{E_{w}}$, so that we can compute a $\delta_{E_{w}}^{\omega}$-name of $\left(x_{n}\right)_{n}$. Thus we can compute $K$ as an element of $\mathscr{V}^{\mathrm{co}}\left(E_{w}\right)$ using Proposition 2.4.

Propositions 4.8 and 2.4 imply that a convex subset of a reflexive computable Banach space $E$ is weakly overt if and only if it has a computable norm-dense sequence.

Finally, we prove a useful uniform characterisation of computably weakly compact convex sets in a reflexive Banach space $E$ with computable dual $E^{\prime}$, which will be an important ingredient for the proof of our main result. Note that by Mazur's lemma, a convex subset of $E$ is weakly compact if and only if it is closed and bounded (cf. also [58, Proposition 2.8.1]).

Definition 4.9. Let $E$ be a reflexive Banach space with computable dual $E^{\prime}$.

(i) A rational half space is a nonempty set of the form

$$
h=\left\{x \in E \mid\left(x, x_{h}^{\prime}\right)+a_{h} \leq 0\right\}
$$

where $x_{h}^{\prime}$ is a rational point in $E^{\prime}$ and $a_{h} \in \mathbb{Q}$. A $\nu_{\mathrm{HB}}$-name of a rational half space is a $\nu_{E^{\prime}} \times \nu_{\mathbb{Q}}$-name of $\left(x_{h}^{\prime}, a_{h}\right) \in E^{\prime} \times \mathbb{Q}$.

(ii) Let $K \subseteq E$ be closed, convex and bounded. A $\kappa_{\mathrm{HB}}$-name of $K$ is a $\nu_{\mathrm{HB}}^{\omega} \times \kappa^{E_{w}}$-name of all rational half spaces containing $K$ in their interior and a weakly compact set $L \in \mathscr{K}\left(E_{w}\right)$ containing $K$.

Note that by Theorem 4.4 (ii), a $\kappa_{\mathrm{HB}}$-name of a closed, convex and bounded set can be computed from a list of all rational half spaces containing $K$ in their interior and a rational bound on $\sup \{\|x\| \mid x \in K\}$. It may not be immediately obvious that $\kappa_{\mathrm{HB}}$ is a welldefined representation. This follows however from the following easy consequence of the Hahn-Banach separation theorem (cf. e.g. [80, Theorem III.2.5]).

Lemma 4.10. Let $E$ be a reflexive Banach space with computable dual $E^{\prime}$. Let $K \subseteq E$ be closed, bounded and convex, let $x \notin K$. Then there exists a rational half space $h$ such that $K \subseteq h^{\circ}$ and $x \in h^{C}$.

Obviously, the boundedness condition on $K$ cannot be dropped, as the example of a straight line with irrational slope in $\mathbb{R}^{2}$ shows.

Theorem 4.11. Let $E$ be a reflexive Banach space with computable dual $E^{\prime}$. Then we have $\left.\kappa_{\mathrm{HB}} \equiv\left(\kappa^{E_{w}}\right)\right|^{\mathscr{K}^{\mathrm{co}}\left(E_{w}\right)}$, where $\mathscr{K}^{\mathrm{co}}\left(E_{w}\right)$ denotes the space of convex weakly compact subsets of $E_{w}$. 
Proof. $\left[\kappa_{\mathrm{HB}} \leq\left.\left(\kappa^{E_{w}}\right)\right|^{\mathscr{K}^{\mathrm{co}}\left(E_{w}\right)}\right]$ : Suppose we are given a $\kappa_{\mathrm{HB}}$-name of $K \in \mathscr{K}^{\mathrm{co}}\left(E_{w}\right)$. Since the name provides us with a weakly compact set $L \in \mathscr{K}\left(E_{w}\right)$ containing $K$, and since id: $\mathscr{A}\left(L_{w}\right) \rightarrow \mathscr{K}\left(L_{w}\right)$ is computable by Proposition 2.8 (i), it suffices to show that we can compute a $\psi^{E_{w}}$-name of $K$. Given $x \in E_{w}$ and a $\nu_{\mathrm{HB}}$-name of a functional $f=\left(\cdot, x_{h}^{\prime}\right)+a_{h}$ we can compute $f(x) \in \mathbb{R}$. Now, if the sequence of half spaces containing $K$ given by the $\kappa_{\mathrm{HB}}$-name is defined by the sequence of affine linear functionals $\left(f_{n}\right)_{n}$, we can compute the characteristic function of $K^{C}$ into Sierpiński-space as follows: given $x \in E_{w}$, if there exists an $n \in \mathbb{N}$ such that $f_{n}(x)>0$ output one, otherwise output zero. This shows that we can compute a $\psi^{E_{w}}$-name of $K$, which proves the claim.

$\left[\left.\left(\kappa^{E_{w}}\right)\right|^{\mathscr{K}^{\mathrm{co}}\left(E_{w}\right)} \leq \kappa_{\mathrm{HB}}\right]:$ Suppose we are given a $\kappa^{E_{w}}$-name of $K$. We need to compute a weakly compact set $L \in \mathscr{K}\left(E_{w}\right)$ with $L \supseteq K$ and a list of all rational half spaces containing $K$ in their interior. Since $K$ contains itself and is given as a $\kappa^{E_{w}}$-name, we may put $L=K$, so that it suffices to show that we can enumerate all rational half spaces containing $K$ in their interior. We show that given a $\nu_{\mathrm{HB}}$-name of an affine linear functional (technically, of the half space defining the functional) $f: E \rightarrow \mathbb{R}$ of the form $f(x)=\left(x, x^{\prime}\right)+a$, we can verify if $f(x)<0$ for all $x \in K$. We can computably translate the $\nu_{\mathrm{HB}}$-name of $f$ into a $\delta_{E^{\prime}}$-name of $x^{\prime}$. Then by definition of $E_{w}$, the mapping $f: E_{w} \rightarrow \mathbb{R}, x \mapsto\left(x, x^{\prime}\right)+a$ is computable. It follows that $U_{f}=\left\{x \in E_{w} \mid f(x)<0\right\}$ is semi-decidable relative to $f$. By definition of $\kappa$, the relation $K \subseteq U_{f}$ is semi-decidable relative to $f$ as well, which proves the claim.

The proof of Theorem 4.11 shows that the definition of $\kappa_{\mathrm{HB}}$ can be slightly relaxed.

Lemma 4.12. Let $E$ be a reflexive Banach space with computable dual $E^{\prime}$. Define a new representation $\tilde{\kappa}_{\mathrm{HB}}$ of $\mathscr{K}^{\mathrm{co}}\left(E_{w}\right)$ as follows: a $\tilde{\kappa}_{\mathrm{HB}}$-name of $K \in \mathscr{K}^{\mathrm{co}}\left(E_{w}\right)$ is a $\nu_{\mathrm{HB}}^{\omega} \times \kappa^{E_{w}}$ name of a sequence $\left(h_{n}\right)_{n}$ of rational half spaces such that $K=\bigcap_{n \in \mathbb{N}} h_{n}^{\circ}$ and a weakly compact set $L \in \mathscr{K}\left(E_{w}\right)$ containing $K$. Then $\tilde{\kappa}_{\mathrm{HB}} \equiv \kappa_{\mathrm{HB}}$.

Proof. Clearly, $\kappa_{\mathrm{HB}} \leq \tilde{\kappa}_{\mathrm{HB}}$. For the converse direction, note that the proof of the reduction $\kappa_{\mathrm{HB}} \leq\left.\left(\kappa^{E_{w}}\right)\right|^{\mathscr{\mathscr { K }}^{\mathrm{co}}\left(E_{w}\right)}$ in Theorem 4.11 actually establishes the stronger reduction $\tilde{\kappa}_{\mathrm{HB}} \leq\left.\left(\kappa^{E_{w}}\right)\right|^{\mathscr{K}^{\mathrm{co}}\left(E_{w}\right)}$, so that we obtain the reduction chain

$$
\tilde{\kappa}_{\mathrm{HB}} \leq\left.\left(\kappa^{E_{w}}\right)\right|^{\mathscr{K}^{\mathrm{co}}\left(E_{w}\right)} \leq \kappa_{\mathrm{HB}}
$$

and thus $\tilde{\kappa}_{\mathrm{HB}} \equiv \kappa_{\mathrm{HB}}$.

On compact subsets of a Banach space $E$, the weak topology and the norm topology coincide. This is effectively witnessed by our representation.

Proposition 4.13. Let $E$ be a reflexive computable Banach space with computable dual, such that the mapping $(\cdot, \cdot)$ is $\left(\delta_{E} \times \delta_{E^{\prime}}, \rho\right)$-computable. Let $K \subseteq E$ be a computably compact and computably overt subset of $E$. Then we have $\left.\left.\delta_{E_{w}}\right|^{K} \equiv \delta_{E}\right|^{K}$.

Proof. Since $(\cdot, \cdot)$ is computable, we have $\delta_{E} \leq \delta_{E_{w}}$, so we only have to show the converse reduction $\left.\delta_{E_{w}}\right|^{K} \leq\left.\delta_{E}\right|^{K}$. Define the represented spaces

$$
K=\left(K,\left.\delta_{E}\right|^{K}\right) \text { and } K_{w}=\left(K,\left.\delta_{E_{w}}\right|^{K}\right) .
$$

Firstly, observe that $E_{w}$ is effectively Hausdorff, i.e. the mapping

$$
E_{w} \rightarrow \mathscr{A}\left(E_{w}\right), x \mapsto\{x\}
$$


is computable: we can verify if two given elements in $E_{w}$ are different by comparing their values on the rational points of $E^{\prime}$. It follows that the identity id: $\mathscr{O}(K) \rightarrow \mathscr{O}\left(K_{w}\right)$ is computable via the following chain of maps:

$$
\begin{gathered}
\mathscr{O}(K) \longrightarrow \mathscr{A}(K) \stackrel{(1)}{\longrightarrow} \mathscr{K}(K) \stackrel{(2)}{\longrightarrow} \mathscr{K}\left(K_{w}\right) \stackrel{(3)}{\longrightarrow} \mathscr{A}\left(K_{w}\right) \longrightarrow K_{w} \longrightarrow \mathscr{O}\left(K_{w}\right) \\
U \longrightarrow K \backslash U \longrightarrow U \longrightarrow U .
\end{gathered}
$$

The computability of (1) follows from the computable compactness of $K$ together with Proposition 2.8 (i). The computability of (2) can be derived from the computability of id: $\mathscr{O}\left(E_{w}\right) \rightarrow \mathscr{O}(E)$, which in turn follows from the computability of id: $E \rightarrow E_{w}$, and the computability of (3) follows from the fact that $E_{w}$ is effectively Hausdorff, together with Proposition 2.8 (ii).

We then obtain the mapping id: $K_{w} \rightarrow K$, i.e. the reduction $\left.\delta_{E_{w}}\right|^{K} \leq\left.\delta_{E}\right|^{K}$, via the following chain of maps:

$$
\begin{gathered}
K_{w} \stackrel{(4)}{\longrightarrow} \mathscr{A}\left(K_{w}\right) \stackrel{(5)}{\longrightarrow} \mathscr{K}\left(K_{w}\right) \stackrel{(6)}{\longrightarrow} \mathscr{K}(K) \stackrel{(7)}{\longrightarrow} K \\
x \longrightarrow\{x\} \longrightarrow\{x\} \longrightarrow x .
\end{gathered}
$$

Mapping (4) is computable since $E_{w}$ is effectively Hausdorff. To establish the computability of (5), observe that the computability of (2) and the computable compactness of $K$ imply that $K \in \mathscr{K}\left(K_{w}\right)$ and apply Proposition 2.8 (i). The computability of (6) follows from the computability of id: $\mathscr{O}(K) \rightarrow \mathscr{O}\left(K_{w}\right)$, which we have established above. For the computability of (7), observe that we can verify if a rational ball of the form $B\left(a, 2^{-n}\right)$ contains $\{x\}$, which yields a Cauchy sequence effectively converging to $x$ by exhaustive search over all rational balls.

Finally, we observe that computably overt, co-semi-decidable subsets of $E_{w}^{\prime}$ are (uniformly) located. The following proposition guarantees that this actually makes sense.

Proposition 4.14. Let $E$ be a Banach space. Let $A \subseteq E$ be weakly sequentially closed. Then $A$ is closed with respect to the norm topology.

Proof. Since $E$ is a metric space, $A$ is closed if and only if it is sequentially closed. Let $\left(x_{n}\right)_{n}$ be a sequence in $A$ with limit $x \in E$. Then $x$ is a weak limit of $\left(x_{n}\right)_{n}$, so $x \in A$, since $A$ is weakly sequentially closed. It follows that $A$ is sequentially closed, and thus closed.

Proposition 4.15. Let $E$ be a reflexive computable Banach space with computable dual, such that the mapping $(\cdot, \cdot)$ is $\left(\delta_{E} \times \delta_{E^{\prime}}, \rho\right)$-computable. Then the canonical embedding $i: \mathscr{A}\left(E_{w}\right) \backslash\{\emptyset\} \rightarrow \mathscr{A}_{\text {dist }_{<}}(E), A \mapsto A$ is computable.

Proof. Given a sequentially weakly closed set $A \in \mathscr{A}\left(E_{w}\right)$, it suffices to show that we can uniformly computably enumerate all closed balls with rational centres and radii contained in the complement $A^{C}$ of $A$. The result then follows from [20, Theorem 3.9 (1)]. The proof of Theorem 4.4 (ii) allows us to uniformly translate a computable number $r \in \mathbb{R}$ into a name of $\bar{B}(0, r)$ as a weakly compact subset of $E_{w}$, i.e. the mapping

$$
(0, \infty) \rightarrow \mathscr{K}\left(E_{w}\right), r \mapsto \bar{B}(0, r)
$$

is computable. It is easy to see that the mapping

$$
E_{w} \times E_{w} \rightarrow E_{w},(x, c) \mapsto x+c
$$


is computable. Hence, the mapping

$$
E_{w} \times(0, \infty) \rightarrow \mathscr{K}\left(E_{w}\right),(c, r) \mapsto \bar{B}(c, r)
$$

is computable. It follows that the mapping

$$
\mathscr{A}\left(E_{w}\right) \times E_{w} \times(0, \infty) \rightarrow \mathbb{S},(A, c, r) \mapsto \begin{cases}1 & \text { if } \bar{B}(c, r) \subseteq A^{C}, \\ 0 & \text { otherwise }\end{cases}
$$

is computable. Since $(\cdot, \cdot)$ is computable, we have $\delta_{E} \leq \delta_{E_{w}}$, so that in particular the mapping

$$
\mathscr{A}\left(E_{w}\right) \times E \times(0, \infty) \rightarrow \mathbb{S},(A, c, r) \mapsto \begin{cases}1 & \text { if } \bar{B}(c, r) \subseteq A^{C}, \\ 0 & \text { otherwise }\end{cases}
$$

is computable. Using this mapping we can enumerate all rational closed balls contained in the complement of $A$.

Proposition 4.15 in particular implies that any nonempty weakly co-semi-decidable subset of $E$ is lower semi-located, and hence every nonempty weakly co-semi-decidable and computably overt subset of $E$ is located (which by Proposition 2.15) is at least not uniformly true for co-semi-decidable subsets of $E$, if $E$ is infinite dimensional).

Let us introduce some further Weihrauch degrees. Let $E$ be a computable Banach space with computable dual, such that the mapping $(\cdot, \cdot)$ is $\left(\delta_{E} \times \delta_{E^{\prime}}, \rho\right)$-computable. Let $A \subseteq E$ be nonempty and weakly closed. The weak closed choice principle $\mathrm{C}_{A}^{\mathrm{w} \rightarrow \mathrm{w}}$ on $A$ is the closed choice principle $\mathrm{C}_{A_{w}}$ on the represented space $A_{w}=\left(A, \delta_{E_{w}}\right)$. The weak-strong closed choice principle is the multimapping

$$
\mathrm{C}_{A}^{\mathrm{w} \rightarrow \mathrm{n}}: \mathscr{A}\left(A_{w}\right) \rightrightarrows A, S \mapsto S,
$$

where the image is represented by $\left.\delta_{E}\right|^{A}$. Similarly, we define $\mathrm{ConvC}_{A}^{\mathrm{w} \rightarrow \mathrm{w}}, \mathrm{UC}_{A}^{\mathrm{w} \rightarrow \mathrm{w}}, \mathrm{ConvC}_{A}^{\mathrm{w} \rightarrow \mathrm{n}}$ and $\mathrm{UC}_{A}^{\mathrm{w} \rightarrow \mathrm{n}}$. We may also define a (computationally) weaker version of the Browder-GöhdeKirk theorem. Let $K$ be nonempty, computably overt, weakly co-semi-decidable, bounded and convex. The weak Browder-Göhde-Kirk theorem is the mapping

$$
\mathrm{WBGK}_{K}: \mathscr{N}(K) \rightrightarrows K_{w}, f \mapsto \operatorname{Fix}(f),
$$

where we are given a nonexpansive mapping like in the case of the Browder-Göhde-Kirk theorem, but are only required to compute a fixed point with respect to the weak topology. Note that in $\ell^{2}$ this amounts to computing a fixed point with respect to an orthonormal basis, but not necessarily computing its $\ell^{2}$-norm (cf. also [9]).

\section{Characterisation of the Fixed Point Sets of Computable Nonexpansive Mappings in Computable Hilbert Space}

We may now prove our main result. Throughout this section we will work on a computable Hilbert space $H$. Note that in this case $H^{\prime} \simeq H$ is again a computable Hilbert space, and that the mapping $(\cdot, \cdot): H \times H^{\prime} \rightarrow \mathbb{R}$ is the usual inner product on $H$, which is computable by the polarisation identity. In particular, we can use Definition 4.1 to construct the space $H_{w}$, whose representation is admissible for the weak topology on $H$.

Theorem 5.1. Let $H$ be a computable Hilbert space, let $K \subseteq H$ be weakly co-semi-decidable, computably overt, bounded, and convex. Then 
(i) The mapping

$$
\text { Fix }: \mathscr{N}(K) \rightarrow \mathscr{K}^{\mathrm{co}}\left(K_{w}\right) \backslash\{\emptyset\}, f \mapsto \operatorname{Fix}(f)
$$

is computable.

(ii) And so is its multivalued inverse

$$
\operatorname{Fix}^{-1}: \mathscr{K}^{\mathrm{co}}\left(K_{w}\right) \backslash\{\emptyset\} \rightrightarrows \mathscr{N}(K) .
$$

Let us sketch the proof of the second claim. Given a nonempty, weakly closed, bounded and convex subset $A$ of $K$, by Theorem 4.11 we can enumerate a sequence of half spaces whose intersection is equal to $A$. Now, the projections onto these half spaces are nonexpansive, thanks to Theorem 2.23 (ii), and computable:

Lemma 5.2. Let $H$ be a computable Hilbert space. There exists a computable function which takes as input a rational half space $h \subseteq H$, encoded as a $\nu_{\mathrm{HB}}-n a m e$, and returns as output the metric projection onto $h$ as an element of $\mathscr{C}(H, H)$.

Proof. Let $h=\left\{x \in H \mid\left(x, x_{h}\right)+a_{h} \leq 0\right\}$, where $x_{h}$ is a rational point in $H$ and $a_{h} \in \mathbb{Q}$. It follows from Lemma 2.17 that we can without loss of generality assume that the set $\left\{n \in \mathbb{N} \mid \nu_{H}(n)=0\right\}$ is decidable (cf. also [12, Lemma 3]). Thus, we can decide if $x_{h}=0$, and if this is the case we necessarily have $a_{h}=0$ (since $h$ is nonempty), and the projection onto $h$ is the identity on $H$. If $x_{h} \neq 0$, put $\tilde{x}_{h}=\frac{x_{h}}{\left\|x_{h}\right\|}, \tilde{a}_{h}=\frac{a_{h}}{\left\|x_{h}\right\|}$, and $p=x-\alpha \tilde{x}_{h}$, where $\alpha=\max \left\{0,\left(x, \tilde{x}_{h}\right)+\tilde{a}_{h}\right\}$. One easily verifies that $p \in h$ and that $p$ satisfies the variational inequality (Theorem 2.23 (ii)). It follows that $P_{h}(x)=p$. This proves the claim.

We can hence compute a sequence of nonexpansive mappings such that $A$ is the intersection of the fixed point sets of these mappings. The following theorem due to Bruck allows us to construct a single nonexpansive mapping whose fixed point set is the intersection of the fixed point sets of our sequence of mappings.

Theorem 5.3 ([27]). Let $E$ be a strictly convex real normed space, let $K \subseteq E$ be nonempty, closed, bounded, and convex. Let $\left(\lambda_{n}\right)_{n}$ be any sequence in $(0,1)$ satisfying $\sum_{n} \lambda_{n}=1$. Let $\left(f_{n}\right)_{n}$ be a family of nonexpansive mappings on $K$ with $\bigcap_{n \in \mathbb{N}} \operatorname{Fix}\left(f_{n}\right) \neq \emptyset$. Then the mapping

$$
f=\sum_{n} \lambda_{n} f_{n}
$$

is well-defined, nonexpansive and satisfies

$$
\operatorname{Fix}(f)=\bigcap_{n \in \mathbb{N}} \operatorname{Fix}\left(f_{n}\right) .
$$

In the final step, we project back onto $K$ in order to construct a self-map of $K$.

Lemma 5.4. Let $H$ be a real Hilbert space, $K \subseteq H$ be closed and convex and $f: K \rightarrow H$ be nonexpansive and suppose that $\operatorname{Fix}(f) \neq \emptyset$. Let $P_{K}$ denote the metric projection onto $K$. Then $P_{K} \circ f$ is nonexpansive as well with $\operatorname{Fix}\left(P_{K} \circ f\right)=\operatorname{Fix}(f)$.

Proof. It is clear that $P_{K} \circ f$ is nonexpansive and that $\operatorname{Fix}(f) \subseteq \operatorname{Fix}\left(P_{K} \circ f\right)$. Suppose there exists $x \in K$ with $f(x) \neq x$ and $P_{K}(f(x))=x$. Let $y$ be some fixed point of $f$. Then

$$
\|f(x)-f(y)\|^{2}=\|y-x\|^{2}+\|x-f(x)\|^{2}-2(f(x)-x, y-x) .
$$

By assumption, $x=P_{K}(f(x)$ ), so by the variational inequality (Theorem 2.23)

$$
(f(x)-x, y-x) \leq 0 \text { for all } y \in K \text {. }
$$


We also assumed that $f(x) \neq x$, i.e. $\|f(x)-x\|^{2}>0$, hence

$$
\|f(x)-f(y)\|^{2}>\|x-y\|^{2} .
$$

Contradicting the assumption that $f$ is nonexpansive.

Proof of Theorem 5.1 (ii). We prove that given a $\kappa_{\mathrm{HB}}$-name $\phi$ of a nonempty, closed, convex subset $A \subseteq K$ we can compute the name of a nonexpansive function $f: K \rightarrow K$ with $\operatorname{Fix}(f)=A$. The name $\phi$ encodes a sequence of rational half spaces $\left(h_{k}\right)_{k}$ containing $A$ in their interior. Using Lemma 5.2 , given $\phi$ we can compute a $\left[\delta_{H} \rightarrow \delta_{H}\right]^{\omega}$-name of some sequence $\left(P_{k}\right)_{k}$ of projections, where $P_{k}$ is the projection onto the rational half space $h_{k}$. By Theorem [5.3, the mapping $g=\sum_{k \in \mathbb{N}} 2^{-k-1} P_{k}$ will satisfy $\operatorname{Fix}(g)=\bigcap_{k \in \mathbb{N}} \operatorname{Fix}\left(P_{k}\right)=A$. By Lemma 5.4, the mapping $P_{K} \circ g: K \rightarrow K$ will have the same set of fixed points. Note that $P_{K}$ is computable by Corollary 2.26, since $K$ is located by Proposition 4.15.

In order to prove item (i) of Theorem 5.1, we need to inspect Lemma 5.4 a little closer. We first need another simple lemma.

Lemma 5.5. Let $E$ be real normed space, let $C \subseteq E$ be closed and convex, let $S \subseteq C$ be a dense subset of $C$ and let $h$ be a half space in $E$. If $C \cap h^{\circ}$ is nonempty, then $S \cap h^{\circ}$ is dense in $C \cap h$.

Proof. Let $x \in C \cap h$, and let $c \in C \cap h^{\circ}$. Then the line segment joining $c$ and $x$ is contained in $C \cap h$ and contains an element $b \in B(x, \varepsilon / 2) \cap h^{\circ}$. Now we choose $a \in S$ in a sufficiently small ball around $b$, so that $d(x, a)<\varepsilon$.

Lemma 5.5 guarantees that intersections of weakly closed and overt sets and rational half spaces are (uniformly) overt. This is a special property, as in general the intersection operator on closed sets is $((\psi \sqcap v) \times(\psi \sqcap v), v)$-discontinuous (cf. [79, Theorem 5.1.13]).

Corollary 5.6. Let $E$ be a computable Banach space with computable dual $E^{\prime}$, such that the mapping $(\cdot, \cdot)$ is $\left(\delta_{E} \times \delta_{E^{\prime}}, \rho\right)$-computable. Then intersection of weakly closed convex sets $C$ and closed rational half spaces $h$ with $C \cap h^{\circ} \neq \emptyset$ is $\left(\left(\psi^{E_{w}} \sqcap v^{E_{w}}\right) \times \nu_{\mathrm{HB}},\left(\psi^{E_{w}} \sqcap v^{E_{w}}\right)\right)$ computable, and hence $\left(\left(\psi^{E_{w}} \sqcap v^{E_{w}}\right) \times \nu_{\mathrm{HB}}, \psi_{\text {dist }}\right)$-computable.

Proof. Since $\nu_{\mathrm{HB}} \leq \psi$, we can always uniformly compute a $\psi$-name of the intersection. In order to compute an $v$-name, enumerate all elements given by the $v$-name of $C$ which are also contained in $h^{\circ}$. The above lemma guarantees that this yields an $v$-name of $C \cap h$. The second claim follows from $\left(\psi^{E_{w}} \sqcap v^{E_{w}}\right) \equiv \psi_{\text {dist }}$, which in turn follows from Proposition 4.15 .

Lemma 5.7. Let $H$ be a real Hilbert space, $K \subseteq H$ be closed, bounded and convex, let $f: K \rightarrow K$ be nonexpansive, $h$ be a half space such that $h^{\circ} \cap K \neq \emptyset$ and let $S \subseteq K$ be dense in $K$. Let $A=h \cap K$. Then $\operatorname{Fix}(f) \cap h=\emptyset$ if and only if

$$
\exists x \in h^{\circ} \cap S . \exists n \in \mathbb{N} .\left(\|f(x)-x\|>2^{-n} \wedge\left\|P_{A}(f(x))-x\right\|<\frac{2^{-2 n-3}}{B}\right),
$$

where $B \geq \sup \{\|x\|+1 \mid x \in K\}$.

Proof. Let us first prove the forward direction. By the Browder-Göhde-Kirk Theorem, $\left.P_{A} \circ f\right|_{A}: A \rightarrow A$ has a fixed point $\tilde{x} \in h \cap K$. Since, by assumption, $\tilde{x}$ is not a fixed point 
of $f$, there exists an $m \in \mathbb{N}$ with $\|f(\tilde{x})-\tilde{x}\|>2^{-m}$. By Lemma 5.5, $S \cap h^{\circ}$ is dense in $h \cap K$, so that we may choose $x \in S \cap h^{\circ}$ with $\|x-\tilde{x}\|<\frac{2^{-2 m-6}}{B}$. Then

$$
\begin{aligned}
\left\|P_{A}(f(x))-x\right\| & \leq\left\|P_{A}(f(\tilde{x}))-\tilde{x}\right\|+\left\|P_{A}(f(x))-P_{A}(f(\tilde{x}))\right\|+\|x-\tilde{x}\| \\
& \leq 2\|x-\tilde{x}\|<\frac{2^{-2 m-5}}{B}=\frac{2^{-2(m+1)-3}}{B} .
\end{aligned}
$$

And (using $B \geq 1$ )

$$
\|f(x)-x\| \geq\|f(\tilde{x})-\tilde{x}\|-2\|x-\tilde{x}\|>2^{-m}-\frac{2^{-2 m-5}}{B} \geq 2^{-m}-2^{-2 m-5}>2^{-m-1} .
$$

For the converse direction, we proceed by contrapositive. We suppose that there exists $y \in \operatorname{Fix}(f) \cap h$ (and hence $y \in \operatorname{Fix}\left(\left.P_{A} \circ f\right|_{A}\right)$ ) and show

$$
\forall x \in h^{\circ} \cap S . \forall n \in \mathbb{N} .\left(\|f(x)-x\|>2^{-n} \rightarrow\left\|P_{A}(f(x))-x\right\| \geq \frac{2^{-2 n-3}}{B}\right) .
$$

Let $x \in h^{\circ} \cap S$ with $\|f(x)-x\|>2^{-n}$. Since $f$ is nonexpansive, we have

$$
\begin{aligned}
\|y-x\|^{2} & \geq\|f(y)-f(x)\|^{2} \\
& =\left\|f(y)-P_{A}(f(x))\right\|^{2}+\left\|P_{A}(f(x))-f(x)\right\|^{2}+2\left(y-P_{A}(f(x)), P_{A}(f(x))-f(x)\right) .
\end{aligned}
$$

Now, by the variational inequality, $2\left(y-P_{A}(f(x)), P_{A}(f(x))-f(x)\right) \geq 0$, so that

$$
\begin{aligned}
\|y-x\|^{2} & \geq\left\|f(y)-P_{A}(f(x))\right\|^{2}+\left\|P_{A}(f(x))-f(x)\right\|^{2} \\
& =\|y-x\|^{2}+\left\|x-P_{A}(f(x))\right\|^{2}+2\left(y-x, x-P_{A}(f(x))\right) \\
& +\left\|P_{A}(f(x))-x\right\|^{2}+\|x-f(x)\|^{2}+2\left(P_{A}(f(x))-x, x-f(x)\right),
\end{aligned}
$$

which entails that

$$
\begin{aligned}
0 & \geq\|f(x)-x\|^{2}+2\left(P_{A}(f(x))-x, x-f(x)-y+x\right) \\
& \geq\|f(x)-x\|^{2}-2\left\|P_{A}(f(x))-x\right\| \cdot\|x-f(x)-y+x\| \\
& \geq\|f(x)-x\|^{2}-8\left\|P_{A}(f(x))-x\right\| B,
\end{aligned}
$$

and hence

$$
\left\|P_{A}(f(x))-x\right\| \geq \frac{2^{-2 n-3}}{B} .
$$

Note that it follows from Corollary 5.6 that the projection onto $A$ in Lemma 5.7 is computable.

Proof of Theorem $5.1(i)$. Given a nonexpansive mapping $f: K \rightarrow K$, we want to compute a $\kappa_{\mathrm{HB}}$-name of $\operatorname{Fix}(f)$. We need to compute a weakly compact set $L \in \mathscr{K}\left(E_{w}\right)$ with $L \supseteq \operatorname{Fix}(f)$, and a list of all rational half spaces containing $f$. Since $K$ contains $\operatorname{Fix}(f)$ and is computably weakly compact by Theorem 4.4 (ii) we may put $L=K$, so it suffices to list all rational half spaces containing $\operatorname{Fix}(f)$. In fact, by Lemma 4.12 it suffices to compute a list of rational half spaces $\left(h_{n}\right)_{n}$ satisfying $\bigcap_{n \in \mathbb{N}} h_{n}^{\circ}=\operatorname{Fix}(f)$. In order to do so, we enumerate two different lists $L_{1}$ and $L_{2}$ of half spaces and interleave them. The first list $L_{1}$ consists of all rational half spaces containing $K$ in their interior. This list is computable since $K$ is computably weakly compact, and hence $\kappa_{\mathrm{HB}}$-computable by Theorem 4.11. In order to compute the second list $L_{2}$, we first enumerate all rational half spaces $h$ such that $h^{\circ} \cap K \neq \emptyset$ and $h^{C} \cap K \neq \emptyset$. This is possible because $K$ is computably overt. Out of these 
half spaces we only enumerate those which satisfy $\operatorname{Fix}(f) \cap\left(h^{\circ}\right)^{C}=\emptyset$. In order to verify this property we apply Lemma 5.7 to the half space $\left(h^{\circ}\right)^{C}$. Note that we can compute the projection onto $K \cap\left(h^{\circ}\right)^{C}$ by Corollary [5.6, so that the property (5.1) in Lemma 5.7 becomes semi-decidable. Now, $\operatorname{Fix}(f) \cap\left(h^{\circ}\right)^{C}=\emptyset$ is equivalent to $h^{\circ} \supseteq \operatorname{Fix}(f)$, and it is easy to see that the list $\left(h_{n}\right)_{n}$ we obtain by interleaving $L_{1}$ and $L_{2}$ satisfies $\bigcap_{n \in \mathbb{N}} h_{n}^{\circ}=\operatorname{Fix}(f)$.

Theorem 5.1 now allows us to determine the Weihrauch degree of the weak and strong Browder-Göhde-Kirk theorem.

Theorem 5.8. Let $H$ be a computable Hilbert space and $K \subseteq H$ be nonempty, bounded, convex, computably weakly closed, and computably overt. Then

$$
\mathrm{BGK}_{K} \equiv{ }_{W} \mathrm{ConvC}_{K}^{\mathrm{w} \rightarrow \mathrm{n}} \text {, }
$$

and

If $K$ is computably compact, then

$$
\mathrm{WBGK} \equiv{ }_{W} \mathrm{ConvC}_{K}^{\mathrm{w} \rightarrow \mathrm{w}} .
$$

$$
\mathrm{BGK}_{K} \equiv_{W} \mathrm{WBGK}_{K} \equiv_{W} \operatorname{ConvC}_{K} \text {. }
$$

Proof. The equivalences $\mathrm{BGK}_{K} \equiv_{W} \operatorname{ConvC}_{K}^{\mathrm{w} \rightarrow \mathrm{n}}$ and $\mathrm{WBGK} \equiv_{W} \mathrm{ConvC}_{K}^{\mathrm{w} \rightarrow \mathrm{w}}$ follow from Theorem 5.1, together with the fact that by Theorem $4.4 K$ is computably weak* compact, so that id : $\mathscr{A}^{\mathrm{co}}(K) \rightarrow \mathscr{K}^{\mathrm{co}}(K)$ is computable. If $K$ is computably compact, then by Proposition 4.13 we have $\left.\left.\delta_{E}\right|^{K} \equiv \delta_{E_{w}}\right|^{K}$, which yields $\mathrm{BGK}_{K} \equiv_{W} \mathrm{WBGK}_{K} \equiv_{W} \operatorname{ConvC}_{K}$ (note that if two representations of the same space are equivalent, then the induced canonical representations of closed sets are - by construction - equivalent as well).

Theorem 5.8 also shows that on a non-compact domain, negative information on the weak closedness of a set is much stronger than negative information on its norm-closedness. We have for instance $\mathrm{BGK}_{B_{\ell^{2}}} \leq_{W} \operatorname{Proj}_{B_{\ell^{2}}} \equiv_{W} \lim$, and so ConvC $\mathrm{B}_{\ell^{2}}^{\mathrm{w} \rightarrow \mathrm{n}} \leq_{W}$ lim, while already $\mathrm{UC}_{B_{\ell^{2}}}^{\mathrm{n} \rightarrow \mathrm{n}}$ is equivalent to the extremely non-effective principle $\mathrm{C}_{\mathbb{N}^{\mathrm{N}}}$. In finite dimension, the degree of $\mathrm{BGK}_{K}$ is always strictly below WKL because of Corollary 3.11. On the unit ball in $\ell^{2}$ this is no longer the case.

Theorem 5.9. There exists a computable mapping

$$
T: \subseteq[0,1]^{\mathbb{N}} \rightarrow \mathscr{K}^{\mathrm{co}}\left(B_{\ell_{w}^{2}}\right)
$$

with $\operatorname{dom} T=\left\{x \in[0,1]^{\mathbb{N}} \mid x(n) \leq x(n+1)\right\}$ such that for all $x \in \operatorname{dom} T$ we have $T(x)=\{a\}$ with $\|a\|_{2}=\lim x(n)$.

Proof. Let $x \in \operatorname{dom} T$. Put $a(0)=x(0)$ and $a(n+1)=\sqrt{x(n+1)^{2}-x(n)^{2}}$. Then we have $a(n)^{2}+\cdots+a(0)^{2}=x(n)^{2}$. Now, put $T(x)=\{a\}$. Note that $a$ is $\delta_{\ell_{w}^{2}}$-computable relative to $x$, so we can compute $\{a\}$ in $\mathscr{K}^{\mathrm{co}}\left(B_{\ell_{w}^{2}}\right)$ : in order to compute the characteristic function of $\{a\}^{C}$ into Sierpiński space we simply check for inequality with $a$ component-wise. This allows us to compute $\{a\}$ as a point in $\mathscr{A}^{\mathrm{co}}\left(B_{\ell_{w}^{2}}\right)$, and thus as a point of $\mathscr{K}^{\mathrm{co}}\left(B_{\ell_{w}^{2}}\right)$, using that the identity id: $\mathscr{A}^{\mathrm{co}}\left(B_{\ell_{w}^{2}}\right) \rightarrow \mathscr{K}^{\mathrm{co}}\left(B_{\ell_{w}^{2}}\right)$ is computable, since $B_{\ell_{w}^{2}}$ is computably weakly compact by Theorem 4.4 . 
Choosing from a set in $\mathscr{K}^{\mathrm{co}}\left(B_{\ell^{2}}\right)$ hence allows us computably translate a $\rho_{<}$-name to a $\rho$-name of a given real number $x \in[0,1]$, already if the set is a singleton. This yields:

Corollary 5.10. $\mathrm{BGK}_{B_{\ell^{2}}} \equiv_{W} \mathrm{UC}_{B_{\ell^{2}}}^{\mathrm{w} \rightarrow \mathrm{n}} \equiv_{W}$ lim. In particular $\mathrm{BGK}_{B_{\ell^{2}}} \equiv_{W} \operatorname{Proj}_{B_{\ell^{2}}}$.

Proof. We have $\mathrm{UC}_{B_{\ell^{2}}}^{\mathrm{w} \rightarrow \mathrm{n}} \leq_{W} \mathrm{ConvC}_{B_{\ell^{2}}}^{\mathrm{w} \rightarrow \mathrm{n}} \equiv_{W} \mathrm{BGK}_{B_{\ell^{2}}}$, the latter by Theorem 5.8. Also, $\mathrm{BGK}_{B_{\ell^{2}}} \leq_{W}$ lim by Proposition 3.5 and Proposition 3.8. It follows from Theorem 5.9 that $\mathrm{UC}_{B_{\ell^{2}}}^{\mathrm{w} \rightarrow \mathrm{n}}$ allows us to determine the limit of any computable monotonically increasing sequence $x \in[0,1]^{\mathbb{N}}$, since

$$
\lim _{n \rightarrow \infty} x(n)=\left\|\mathrm{UC}_{B_{\ell^{2}}}^{\mathrm{w} \rightarrow \mathrm{n}}(T(x))\right\|_{2}
$$

and $\|\cdot\|_{2}$ is $\left(\delta_{\ell^{2}}, \rho\right)$-computable. We hence have $\lim \leq_{W} \mathrm{UC}_{B_{\ell^{2}}}^{\mathrm{w} \rightarrow \mathrm{n}}$, which finishes the proof.

In particular we have the following non-uniform corollary:

Corollary 5.11. There exists a computable nonexpansive self-map of the closed unit ball in $\ell^{2}$ with a unique fixed point, which is uncomputable.

Compare Corollary 5.11 to Theorem 3.15: on a compact domain, any computable function without computable fixed points necessarily has uncountably many fixed points, since otherwise it has at least one isolated fixed point which is then computable by Theorem 2.10. If we drop compactness, even unique solutions may be uncomputable. Note however, that since the unit ball in $\ell^{2}$ is still computably weakly compact, unique fixed points on $B_{\ell^{2}}$ are still "weakly computable", in the sense that they are computable as elements in the represented space $\ell_{w}^{2}$. In particular, their coordinates with respect to an orthonormal basis are still computable.

On a computably compact domain, the Weihrauch degree of the theorem is still at most WKL. We can now show that it is in fact equivalent to WKL on the Hilbert cube. In order to do so, we will first have to define the parallelisation of Weihrauch degrees, which was introduced in [17].

Definition 5.12. Let $f: \subseteq X \rightrightarrows Y$ be a partial multimapping. The parallelisation $\hat{f}$ of $f$ is the multimapping $\hat{f}: \subseteq X^{\mathbb{N}} \rightrightarrows Y^{\mathbb{N}}, \hat{f}(\lambda n . x(n))=\lambda n . f(x(n))$.

It is not hard to see that $f \leq_{W} g$ implies $\hat{f} \leq_{W} \hat{g}$ (cf. also Proposition 4.2 in [17]). The following theorem is essentially due to [17] (cf. also Theorem 6.2 and the subsequent comment in [16]).

Theorem 5.13. $\widehat{\mathrm{IVT}} \equiv_{W} \mathrm{WKL}$.

Theorem 5.14. Let $\mathscr{H}=\left\{\sum_{i \in \mathbb{N}} \alpha_{i} e_{i} \mid \alpha_{i} \in\left[0,2^{-i}\right]\right\}$ be the Hilbert cube in $\ell^{2}$. Then $\mathrm{BGK}_{\mathscr{H}} \equiv_{W}$ WKL.

Proof. Clearly, $\mathscr{H}$ is computably compact, so BGK $\mathscr{H}_{W} \equiv_{W}$ ConvC $_{\mathscr{H}} \leq_{W} \mathrm{C}_{\mathscr{H}} \equiv_{W}$ WKL. In order to prove the converse direction, we show that $\widehat{\mathrm{IVT}} \leq_{W} \mathrm{ConvC}_{\mathscr{H}}$. Since $\widehat{\mathrm{IVT}} \equiv_{W} \mathrm{WKL}$ and ConvC ${ }_{\mathscr{H}} \equiv_{W} \mathrm{BGK}_{\mathscr{H}}$, it follows that WKL $\leq_{W}$ BGK $\mathscr{H}$. By Proposition 3.21 we have $\operatorname{IVT} \equiv_{W} \operatorname{ConvC}_{[0,1]}$ and so $\widehat{\mathrm{IVT}} \equiv_{W} \widehat{\operatorname{ConvC}_{[0,1]}}$. Let $\left(\left[a_{n}, b_{n}\right]\right)_{n}$ be a sequence of closed intervals in $\left(\mathscr{A}^{\mathrm{co}}([0,1])\right)^{\mathbb{N}}$. Consider the set $A=\left\{\sum_{i \in \mathbb{N}} \alpha_{i} e_{i} \mid \alpha_{i} \in\left[a_{i} 2^{-i}, b_{i} 2^{-i}\right]\right\} \subseteq \mathscr{H}$. Then $A$ is computable as a point in $\mathscr{K}^{\mathrm{co}}(\mathscr{H})$ relative to $\left(\left[a_{n}, b_{n}\right]\right)_{n}$. Clearly, choosing a point in $A$ allows us to choose a point in $\left(\left[a_{n}, b_{n}\right]\right)_{n}$, so $\widehat{\operatorname{ConvC}_{[0,1]}} \leq{ }_{W} \operatorname{ConvC}_{\mathscr{H}} \equiv_{W} \mathrm{BGK}_{\mathscr{H}}$. 
Theorem 5.14 can (essentially) be viewed as a uniform strengthening of Theorem 3.15. Notice that the proof of Theorem 3.15 can be utilized to establish the reduction $\widehat{\mathrm{LLPO}} \leq_{W} \mathrm{BGK}_{\mathscr{H}}$, which yields a slightly different proof of Theorem $\left[5.14\right.$, since $\widehat{\mathrm{LLPO}} \equiv_{W}$ WKL (again, cf. [17]). The proof of Theorem [5.14 can also be used to show WBGK $B_{\ell^{2}} \equiv_{W}$ WKL. We now have a fairly good idea of the computational content of the Browder-GöhdeKirk theorem. It follows from [55] that $\left(\mathrm{BGK}_{[0,1]^{n}}\right)_{n \in \mathbb{N}}$ is a strictly increasing sequence of Weihrauch degrees, all strictly below WKL. On the compact but infinite dimensional Hilbert cube $\mathscr{H}$ the theorem becomes equivalent to WKL. If we drop compactness and consider the theorem on the unit ball in $\ell^{2}$, it becomes even more non-effective, and in particular equivalent to computing rates of convergence for fixed point iterations, but is still much more effective than full choice on $B_{\ell^{2}}$.

In finite dimension, a computable nonexpansive self-map of a computably compact domain always has computable fixed points by Theorem 3.10, and this relies solely on the fact that the fixed point set is convex. This is reminiscent of the fact that unique zeroes of computable functions are always (in this case even uniformly) computable. A typical feature of such results is that they assert the existence of computable objects, but the computational complexity of these objects is unbounded. This is also the case here: using similar techniques as in [41, we can strengthen Theorem 5.1 ( $(\mathrm{ii})$ to assert for every nonempty co-semi-decidable and convex $A \subseteq K$ the existence of a polynomial-time computable nonexpansive $f: K \rightarrow K$ such that $\operatorname{Fix}(f)=A$, at least in the case where $K$ is computably compact, and so in particular in the finite-dimensional case (if $K$ is not computably compact there is no uniform majorant on the names of the points in $K$, so one would have to work in the framework of second-order complexity [39]). This allows us to characterise the computational complexity of fixed points of Lipschitz-continuous polynomial-time computable functions according to their Lipschitz constant.

Theorem 5.15. Let $[0,1]^{2}$ be the unit square in Euclidean space $\mathbb{R}^{2}$. Let $f:[0,1]^{2} \rightarrow[0,1]^{2}$ be polynomial-time computable and Lipschitz-continuous with Lipschitz constant L. Then:

- If $L<1, f$ has a unique polynomial time computable fixed point, which is uniformly computable relative to the promise that $L<1$ and uniformly polynomial time computable relative to the promise that $L<1-\varepsilon$ for some fixed $\varepsilon>0$.

- If $L=1$, the fixed point set of $f$ can be any nonempty co-semi-decidable convex subset of $[0,1]^{2}$. The multi-valued operator mapping $f$ to some fixed point is realiser-discontinuous and hence uncomputable, but $f$ still has computable fixed points. However, there is no computable bound on the computational complexity of the fixed points of $f$.

- If $L>1, f$ may not have any computable fixed points.

The third claim in Theorem 5.15 follows from a strengthening of the results in [19, which the authors of that paper have recently obtained, but which seems to be unpublished as of yet.

\section{Further Results and Possible Generalisations}

The special case of Theorem 5.1 where the underlying Hilbert space is two-dimensional seems to generalise to uniformly convex and smooth real Banach spaces of dimension two. Note that the first item of the theorem becomes trivial in finite dimension. A Banach space is called smooth, if its dual space is strictly convex and uniformly smooth if its dual space is uniformly convex. For instance, all $L^{p}$-spaces with $1<p<\infty$ are uniformly convex 
and uniformly smooth. The two notions of smoothness and uniform smoothness coincide in finite dimension.

Conjecture 6.1. Let $E$ be a uniformly convex, smooth, computable Banach space of dimension two, and let $K \subseteq E$ be bounded, convex, and located5. Then the multi-valued mapping

is computable.

$$
\operatorname{Fix}^{-1}: \mathscr{K}^{\mathrm{co}}(K) \backslash\{\emptyset\} \rightrightarrows \mathscr{N}(K)
$$

The proof of this result would be almost identical to that of Theorem 5.1. The only places where we used that the underlying space is a Hilbert space were Theorem 2.23, which asserts that the projection onto each convex, closed set is nonexpansive, and Lemma 5.4. In general the projection onto a closed and convex subset of a Banach space will not be nonexpansive. In fact, this property characterises Hilbert spaces (cf. 65]). However, we only need the existence of a computable nonexpansive retraction onto each located convex subset. A retraction $Q: E \rightarrow K$ of $E$ onto a nonempty subset $K \subseteq E$ is called sunny, if

$$
Q(\alpha x+(1-\alpha) Q(x))=Q(x) \text { for all } x \in E, \alpha \in[0,1]
$$

Geometrically, this means that for all $x \notin K$, all points on the ray defined by $x$ and $Q(x)$ with initial point $Q(x)$ are mapped onto the same point $Q(x)$. It is well known that in a smooth Banach space of dimension two, sunny nonexpansive retractions onto closed convex subsets exist and are unique. Consequently, they are computable.

Theorem 6.2 ([38]). Let $E$ be a smooth real Banach space of dimension two. Then for every nonempty closed convex subset $C$ of $E$, there exists a nonexpansive sunny retraction of $E$ onto $C$.

Theorem 6.3 ([26]). Let $E$ be a smooth real Banach space. Let $K \subseteq C$ be two nonempty, closed, and convex subsets of $E$. Then there exists at most one sunny nonexpansive retraction of $C$ onto $K$.

Theorem 6.4. Let $E$ be a smooth computable Banach space of dimension two. Let $C \subseteq E$ be nonempty, convex, bounded, and located. Then the mapping

$$
\text { SRet: } \mathscr{A}_{\text {dist }}^{\mathrm{co}}(C) \backslash\{\emptyset\} \mapsto \mathscr{N}(C)
$$

that maps $K$ to the unique sunny nonexpansive retraction of $C$ onto $K$, is computable.

Proof (sketch). The set of nonexpansive self-maps of $C$ is computably compact, since it is equicontinuous and $C$ is compact. We can verify if a given map $f: C \rightarrow C$ does not leave all points of $K$ fixed, if it maps a point of $C$ to a point outside of $K$, and if $f(\alpha x+(1-\alpha) f(x)) \neq f(x)$ for some $x \in C, \alpha \in[0,1]$. It follows that the set of sunny nonexpansive retractions of $C$ onto $K$ is co-semi-decidable relative to (a $\psi_{\text {dist-name of }} K$. Theorems 6.2 and 6.3 assert that it is a singleton. It follows that the operator is uniformly computable.

\footnotetext{
${ }^{5}$ Recall that in finite dimension a nonempty closed set is located if and only if it is co-semi-decidable and computably overt (cf. also [79).
} 
The other result that relies on Hilbert space techniques is Lemma 5.4, which uses the nonexpansiveness of the projection and the variational inequality. In principle we could replace the projection by the sunny nonexpansive retraction onto the domain, but the question remains whether this will always leave the fixed point set unchanged.

Conjecture 6.5. Let $E$ be a smooth and uniformly convex Banach space of dimension two, let $K \subseteq E$ be nonempty, closed, bounded, and convex and let $f: K \rightarrow E$ be nonexpansive with $\operatorname{Fix}(f) \neq \emptyset$. Let $P: E \rightarrow K$ be the sunny nonexpansive retraction onto $K$. Then we have $\operatorname{Fix}(P \circ f)=\operatorname{Fix}(f)$.

Proof of to Conjecture 6.1 up to Conjecture 6.5. We could now prove Conjecture 6.1 analogously to Theorem 5.1; we are given a convex, closed subset $A$ of $K$ as a $\kappa_{\mathrm{HB}}$-name and want to construct a nonexpansive mapping $f: K \rightarrow K$ with $\operatorname{Fix}(f)=A$. Let $\left(h_{n}\right)_{n}$ be the sequence of half spaces given by the $\kappa_{\mathrm{HB}}$-name. Since $h_{n} \cap K \neq \emptyset$ for all $n$, we can compute a $\psi_{\text {dist }}^{\omega}$-name of the sequence $\left(h_{n} \cap K\right)_{n \in \mathbb{N}}$ thanks to Corollary 5.6. Now, Theorem 6.4 allows us to compute a $\left[\delta_{K} \rightarrow \delta_{K}\right]^{\omega}$-name of the sequence $\left(f_{n}\right)_{n}$ of sunny nonexpansive retractions of $K$ onto $h_{n} \cap K$. Applying Theorem 5.3, we obtain a nonexpansive mapping $g: K \rightarrow E$ with $\operatorname{Fix}(g)=\bigcap_{n \in \mathbb{N}} h_{n}=A$. Finally, we use the computable nonexpansive sunny retraction onto $K$ and Conjecture 6.5 to obtain a self-map $f$ of $K$ with $\operatorname{Fix}(f)=A$.

The only "missing piece" in this proof is Conjecture 6.5. By replacing this conjecture by a weaker statement that we can prove, we obtain a weaker version of Conjecture 6.1, which is almost as good.

Lemma 6.6. Let $E$ be a uniformly convex Banach space of dimension two, let $K \subseteq E$ be nonempty, closed, bounded, and convex. Suppose that $K^{\circ}$ is nonempty and that $\partial K$ does not contain any line segments, and let $f: K \rightarrow E$ be nonexpansive with $\operatorname{Fix}(f) \neq \emptyset$. Let $P: E \rightarrow K$ be the sunny nonexpansive retraction onto $K$. Then $P \circ f$ is nonexpansive as well with $\operatorname{Fix}(P \circ f)=\operatorname{Fix}(f)$.

Proof. Clearly, $P \circ f$ is nonexpansive with $\operatorname{Fix}(f) \subseteq \operatorname{Fix}(P \circ f)$. Suppose that there exists $x \in \operatorname{Fix}(P \circ f)$, which is not a fixed point of $f$. Since $P$ is sunny, $x \in \partial K$. Let $y \in \operatorname{Fix}(f)$. Since $\operatorname{Fix}(f)$ is closed, there exists $\varepsilon>0$ such that $B(x, \varepsilon) \subseteq \operatorname{Fix}(f)^{C}$. Since $P \circ f$ is nonexpansive, the line segment $L$ joining $y$ and $x$ is contained in $\operatorname{Fix}(P \circ f)$. By hypothesis, the line segment without its endpoints has to lie in $K^{\circ}$ (it is easy to see that if a convex set contains three points of a line segment in its boundary, it contains the whole line segment in its boundary). Hence, there exists $z \in K^{\circ} \cap L \cap B(x, \varepsilon)$. Contradiction.

Remark 6.7. A similar proof shows that we may replace the condition that $\partial K$ contains no line segments, by the condition that $\operatorname{Fix}(f) \cap K^{\circ} \neq \emptyset$. In this case we do not even require the retraction to be sunny.

Theorem 6.8. Let $E$ be a uniformly convex, smooth computable Banach space of dimension two, and let $K \subseteq E$ be nonempty, bounded, convex, and located. Suppose that either $\operatorname{dim} K=2$ and $\partial K$ contains no line segments or $\operatorname{dim} K=1$. then the multi-valued mapping

$$
\operatorname{Fix}^{-1}: \mathscr{K}^{\mathrm{co}}(K) \backslash\{\emptyset\} \rightrightarrows \mathscr{N}(K)
$$

is computable.

In a uniformly convex space the unit ball contains no line segments, so $B_{E}$ is an example of an admissible domain $K$. In particular, every co-semi-decidable, convex subset of $B_{E}$ is the fixed point set of some computable, nonexpansive self-map of $B_{E}$. 
Proof of Theorem 6.8. If $\operatorname{dim} K=1$, we introduce suitable coordinates in which $K$ is contained in the $x$-axis and use the construction of Theorem 3.17. If $\operatorname{dim} K=2$, we use the proof of Conjecture 6.1. Note that here we may replace Conjecture 6.5 by Lemma 6.6, so the proof is complete.

The obvious question at this point is whether Conjecture 6.1 might generalise to higher dimensional Banach spaces. While most of the results we used in the proof at least generalise to finite-dimensional smooth and uniformly convex computable Banach spaces the main obstruction appears to be the existence of nonexpansive retractions. Our proof uses the fact that there exist nonexpansive retractions onto every rational half space, but if $E$ is a Banach space of dimension at least three and there exist nonexpansive retractions onto each two-dimensional subspace, then $E$ is a Hilbert space. Similarly, the unit ball of an at least three-dimensional Banach space $E$ is a nonexpansive retract of $E$ if and only if $E$ is a Hilbert space (cf. 25]). On the other hand, every fixed point set of a nonexpansive mapping $f: K \rightarrow K$ is a nonexpansive retraction of $K$. In view of these results it seems likely that Theorem 5.1 characterises computable Hilbert space of dimension three or higher.

Finally, we extend the stronger upper bound obtained in Proposition 3.8 for compact sets and Hilbert space to the noncompact case in uniformly convex and uniformly smooth spaces. For this we need a generalisation of Theorem 1.2 due to Reich [67. We will only state a special case.

Theorem 6.9 (67]). Let $E$ be a uniformly smooth, uniformly convex Banach space, let $K \subseteq E$ be nonempty, closed, bounded and convex, let $f: K \rightarrow K$ be nonexpansive, and let $x \in K$. Put $\alpha_{n}=1-(n+2)^{-\frac{1}{2}}$. Then the sequence $\left(x_{n}\right)_{n}$ defined by the iteration scheme $x_{0}=x$ and

$$
x_{n+1}=\left(1-\alpha_{n}\right) x_{0}+\alpha_{n} f\left(x_{n}\right)
$$

converges to a fixed point of $f$.

Note that the iteration defined in Theorem 6.9 converges to a retraction onto the fixed point set of $f$. In fact, one can show that the sequence $\left(x_{n}\right)_{n}$ converges to $Q\left(x_{0}\right)$, where $Q$ is the unique sunny nonexpansive retraction of $K$ onto $\operatorname{Fix}(f)$.

Theorem 6.10. Let $E$ be a uniformly convex, uniformly smooth computable Banach space. Let $K \subseteq E$ be nonempty, bounded, convex, co-semi-decidable, and computably overt. Then

$$
\operatorname{Proj}_{K} \leq_{W} \lim .
$$

Proof. We use similar ideas as in the proof of Proposition 3.8. Again we exploit the fact that we can actually compute countably many instances of lim in parallel. As in the proof of the general upper bound in Proposition 3.8, we use countably many instances of lim to obtain a function $\mu: \mathbb{N} \rightarrow \mathbb{N}$ satisfying $2^{-\mu(n)} \leq \eta_{E}\left(2^{-n}\right)$, where $\eta_{E}$ is a modulus of uniform convexity for $E$, and another batch of countably many instances to obtain an approximation to the distance function to $\operatorname{Fix}(f)$ from below. Since $K$ is computably overt, it contains a computable dense sequence $\left(x_{n}\right)_{n}$. Let $x_{n}^{0}=x_{n}$ and $x_{n}^{k+1}=\left(1-\alpha_{k}\right) x_{n}^{0}+\alpha_{k} f\left(x_{n}^{k}\right)$ with $\alpha_{k}$ as in Theorem 6.9. Using another countable batch of instances of lim, we obtain the sequence $\left(\lim _{k \rightarrow \infty} x_{n}^{k}\right)_{n}$, which is dense in $\operatorname{Fix}(f)$, since the iteration defines a retraction of $K$ onto $\operatorname{Fix}(f)$. Using this sequence we can compute the distance function to $\operatorname{Fix}(f)$ from above, so that we obtain the distance function to $\operatorname{Fix}(f)$ as an element of $\mathscr{C}(K, \mathbb{R})$. Together with Corollary 2.25 this establishes the reduction. 


\section{ACKNOWLEDGEMENTS.}

The present work was motivated by a question by Ulrich Kohlenbach, whether the Krasnoselski-Mann iteration has nonuniformly computable rates of convergence. He has also provided many valuable insights both concerning fixed point theory and computability theory. This work has greatly benefited from discussions with Vasco Brattka, Arno Pauly, Guido Gherardi, and Martin Ziegler. I would also like to thank the anonymous referees for pointing out many shortcomings in the original version of this paper.

\section{REFERENCES}

[1] S. Arora and B. Barak. Computational Complexity: A Modern Approach. Cambridge University Press, 2009.

[2] J. Avigad, P. Gerhardy, and H. Towsner. Local stability of ergodic averages. Trans. Amer. Math. Soc., 362(1):261-288, 2010.

[3] J. Avigad and J. Rute. Oscillation and the mean ergodic theorem for uniformly convex Banach spaces. Ergodic Theory and Dynamical Systems, Available on CJO 2014, doi:10.1017/etds.2013.90, 2014.

[4] G. Baigger. Die Nichtkonstruktivität des Brouwerschen Fixpunktsatzes. Archiv für Mathematische Logik und Grundlagenforschung, 25:183-188, 1985.

[5] M. Beeson. Foundations of Constructive Mathematics. Springer-Verlag, New York, 1985.

[6] E. Bishop and D. Bridges. Constructive Analysis. Springer-Verlag, 1985.

[7] F. F. Bonsall. Lectures on some fixed point theorems of functional analysis, volume 26 of Tata Institute of Fundamental Research Lectures on Mathematics and Physics: Mathematics. Tata Institute of Fundamental Research, 1962.

[8] V. Brattka. Computing uniform bounds. Electronic Notes in Theoretical Computer Science, 66(1):13 24, 2002. CCA 2002, Computability and Complexity in Analysis.

[9] V. Brattka. Computability on non-separable Banach spaces and Landau's theorem. In L. Crosilla and P. Schuster, editors, From Sets and Types to Topology and Analysis: Towards Practicable Foundations for Constructive Mathematics, pages 316-333. Oxford University Press, 2005.

[10] V. Brattka. Effective Borel measurability and reducibility of functions. Mathematical Logic Quarterly, 51(1):19-44, 2005.

[11] V. Brattka. Computable versions of the uniform boundedness theorem. In Z. Chatzidakis, P. Koepke, and W. Pohlers, editors, Logic Colloquium 2002, volume 27 of Lecture Notes in Logics, pages 130-151. Association for Symbolic Logic, 2006.

[12] V. Brattka. Borel complexity and computability of the Hahn-Banach Theorem. Archive for Mathematical Logic, 46(7-8):547-564, 2008.

[13] V. Brattka, M. de Brecht, and A. Pauly. Closed choice and a uniform low basis theorem. Annals of Pure and Applied Logic, 163(8):986 - 1008, 2012.

[14] V. Brattka and R. Dillhage. Computability of finite dimensional linear subspaces and best approximation. Annals of Pure and Applied Logic, 162:182-193, 2010.

[15] V. Brattka and G. Gherardi. Borel complexity of topological operations on computable metric spaces. Journal of Logic and Computation, 19(1):45-76, 2009.

[16] V. Brattka and G. Gherardi. Effective choice and boundedness principles in computable analysis. Bulletin of Symbolic Logic, 17(1):73-117, 2011.

[17] V. Brattka and G. Gherardi. Weihrauch degrees, omniscience principles and weak computability. J. Symbolic Logic, 76(1):143-176, 2011.

[18] V. Brattka, G. Gherardi, and A. Marcone. The Bolzano-Weierstrass Theorem is the Jump of Weak König's Lemma. Annals of Pure and Applied Logic, 163(6):623-655, 2012.

[19] V. Brattka, S. Le Roux, and A. Pauly. Connected Choice and the Brouwer Fixed Point Theorem. arXiv:1206.4809v1, 2012.

[20] V. Brattka and G. Presser. Computability on subsets of metric spaces. Theoretical Computer Science, $305(1-3): 43-76,2003$. 
[21] V. Brattka and M. Schröder. Computing with sequences, weak topologies and the axiom of choice. In L. Ong, editor, Computer Science Logic, volume 3634 of Lecture Notes in Computer Science, pages 462-476. Springer Berlin Heidelberg, 2005.

[22] L. Brouwer. An intuitionist correction of the fixed point theorem on the sphere. Proceedings of the Royal Societey. London. Series A., 213:1-2, 1952.

[23] F. Browder. Fixed point theorems for noncompact mappings in Hilbert space. Proc. Nat. Acad. Sci. USA, 43:1272-1276, 1965.

[24] F. Browder. Nonexpansive nonlinear operators in a Banach space. Proc. Nat. Acad. Sci. USA, 54:10411044, 1965.

[25] R. Bruck. A characterisation of Hilbert space. Proc. Amer. Math. Soc., 43:173-175, 1974.

[26] R. E. Bruck. Nonexpansive projections on subsets of Banach spaces. Pacific Journal Of Mathematics, 47:341-355, 1973.

[27] R. E. Bruck. Properties of fixed-point sets of nonexpansive mappings in Banach spaces. Trans.Amer.Math.Soc., 179:251-262, 1973.

[28] C. Chidume and S. Mutangadura. An example on the Mann iteration method for Lipschitz pseudocontractions. Proceedings of The American Mathematical Society, 129(8):2359-2363, 2001.

[29] J. A. Clarkson. Uniformly convex spaces. Trans. Amer. Math. Soc., 40:396-414, 1936.

[30] R. DeMarr. Common fixed points for commuting contraction mappings. Pacific J. Math., 13(4):1139$1141,1962$.

[31] M. Escardó. Synthetic topology of data types and classical spaces. Electronic Notes in Theoretical Computer Science, 87, 2004.

[32] K. Goebel. An elementary proof of the fixed-point theorem of Browder and Kirk. The Michigan Mathematical Journal, 16(4):381-383, 121969.

[33] K. Goebel and W. Kirk. Topics in metric fixed point theory. Cambridge University Press, 1990.

[34] D. Göhde. Zum Prinzip der kontraktiven Abbildung. Math. Nachr., 30:251-258, 1965.

[35] B. Halpern. Fixed points of nonexpanding maps. Bull. Amer. Math. Soc., 73(6):957-961, 1967.

[36] A. Hatcher. Algebraic Topology. Cambridge University Press, 2002.

[37] M. D. Hirsch, C. H. Papadimitriou, and S. A. Vavasis. Exponential lower bounds for finding Brouwer fixed points. Journal of Complexity, 5:379-416, 1989.

[38] L. Karlovitz. The construction and application of contractive retractions in 2-dimensional normed linear spaces. Indiana Univ. Math. J., 22:473-481, 1972.

[39] A. Kawamura and S. Cook. Complexity theory for operators in analysis. ACM Trans. Comput. Theory, 4(2):5:1-5:24, May 2012.

[40] W. Kirk. A fixed point theorem for mappings which do not increase distance. Amer. Math. Monthly, 72:1004-1006, 1965.

[41] K.-I. Ko. Complexity Theory of Real Functions. Birkhäuser, 1991.

[42] U. Kohlenbach. New effective moduli of uniqueness and uniform a-priori estimates for constants of strong unicity by logical analysis of known proofs in best approximation theory. Numer. Funct. Anal. Optim., 14:581-606, 1993.

[43] U. Kohlenbach. On the computational content of the Krasnoselski and Ishikawa fixed point theorems. In J. Blanck, V. Brattka, and P. Hertling, editors, Proc. of the Fourth Workshop on Computability and Complexity in Analysis, pages 119-145. Spinger Lecture Notes in Computer Science LNCS 2064, 2001.

[44] U. Kohlenbach. A quantitative version of a theorem due to Borwein-Reich-Shafrir. Numer. Funct. Anal. Optim., 22:641-656, 2001.

[45] U. Kohlenbach. Some computational aspects of metric fixed point theory. Nonlinear Anal., 61:823-837, 2005.

[46] U. Kohlenbach. Applied Proof Theory: Proof Interpretations and their Use in Mathematics. Springer, 2008.

[47] U. Kohlenbach and L. Leustean. Mann iterates of directionally nonexpansive mappings in hyperbolic spaces. Abstr. Appl. Anal., 2003(8):449-477, 2003.

[48] U. Kohlenbach and P. Safarik. Fluctuations, effective learnability and metastability in analysis. Annals of Pure and Applied Logic, 165(1):266-304, 2014.

[49] K. Königsberger. Analysis 2. Springer, 2004.

[50] D. Körnlein and U. Kohlenbach. Rate of metastability for Bruck's iteration of pseudocontractive mappings in Hilbert space. Numer. Funct. Anal. Optim., 35:20-31, 2014. 
[51] M. Krasnoselski. Two remarks on the method of successive approximations. Uspekhi Mat. Nauk, 10:123127, 1955. (Russian).

[52] V. Kreinovich. Categories of space-time models. PhD thesis, Soviet Academy of Sciences, Novosibirsk, 1979. (Russian).

[53] G. Kreisel. On the interpretation of non-finitist proofs, part I. J. Symb. Log., 16:241-267, 1951.

[54] G. Kreisel. On the interpretation of non-finitist proofs, part II: Interpretation of number theory, applications. J. Symb. Log., 17:43-58, 1952.

[55] S. Le Roux and A. Pauly. Closed choice for finite and for convex sets. In P. Bonizzoni, V. Brattka, and B. Löwe, editors, The Nature of Computation. Logic, Algorithms, Applications, volume 7921 of Lecture Notes in Computer Science, pages 294-305. Springer Berlin Heidelberg, 2013.

[56] S. Le Roux and M. Ziegler. Singular coverings and non-uniform notions of closed set computability. Mathematical Logic Quarterly, 54(5):545-560, 2008.

[57] L. Leustean. A quadratic rate of asymptotic regularity for CAT(0)-spaces. J. Math. Anal. Appl., 325:386399, 2007.

[58] R. E. Megginson. An introduction to Banach space theory, volume 183. Springer, 1998.

[59] J. Miller. $\Pi_{1}^{0}$-classes in computable analysis and topology. PhD thesis, Cornell University, 2002.

[60] J. R. Munkres. Topology. Prentice Hall, 2000.

[61] E. Neumann. Computational Problems in Metric Fixed Point Theory and their Weihrauch Degrees. Master's thesis, Technische Universität Darmstadt, August 2014.

[62] Z. Opial. Weak convergence of the sequence of successive approximations for nonexpansive mappings. Bull. Amer. Math. Soc., 73(4):591-597, 1967.

[63] V. Orevkov. A constructive map of the square onto itself, which moves every constructive point. Dokl. Akad. Nauk SSSR, 152:55-58, 1963.

[64] A. Pauly. A new introduction to the theory of represented spaces. arXiv:1204.3763v2, 2013.

[65] R. Phelps. Convex sets and nearest points. Proc. Amer. Math. Soc., 8:790-797, 1957.

[66] M. B. Pour-El and J. I. Richards. Computability in Analysis and Physics. Springer, 1989.

[67] S. Reich. Strong convergence theorems for resolvents of accretive operators in Banach spaces. Journal of Mathematical Analysis and Applications, 75(1):287-292, 1980.

[68] H. Scarf. The approximation of fixed points of a continuous mapping. SIAM J. Appl. Math., 15(5):1328$1343,1967$.

[69] K. Schade and U. Kohlenbach. Effective metastability for modified Halpern iterations in CAT(0) spaces. Fixed Point Theory and Applications, 2012(1), 2012.

[70] M. Schröder. Admissible Representations for Continuous Computations. PhD thesis, FernUniversität Hagen, 2002.

[71] M. Schröder. Extended admissibility. Theoretical Computer Science, 284:519-538, 2002.

[72] E. Specker. Nicht konstruktiv beweisbare Sätze der Analysis. J. Symbolic Logic, 14:145-208, 1949.

[73] E. Specker. Der Satz vom Maximum in der rekursiven Analysis. In A. Heyting, editor, Constructivity in Mathematics, pages 254-265. Studies in Logic and The Foundations of Mathematics, 1959.

[74] L. A. Steen and J. A. Seebach, Jr. Counterexamples in Topology. Springer Verlag, New York, 1978.

[75] T. Tao. Soft analysis, hard analysis, and the finite convergence principle. available online at: http://terrytao.wordpress.com/2007/05/23/soft-analysis-hard-analysis-and-the-finite-convergenceprinciple/, May 2007.

[76] T. Tao. Norm convergence of multiple ergodic averages for commuting transformations. Ergodic Theory and Dynamical Systems, 28:657-688, 42008.

[77] P. Taylor. A lambda calculus for real analysis. Journal of Logic $\&$ Analysis, 2(5):1-115, 2010.

[78] K. Weihrauch. The degrees of discontinuity of some translators between representations of the real numbers. Technical Report TR-92-050, International Computer Science Institute, Berkeley, 1992.

[79] K. Weihrauch. Computable Analysis. Springer, 2000.

[80] D. Werner. Funktionalanalysis. Springer, 2011.

[81] R. Wittmann. Approximation of fixed points of nonexpansive mappings. Archiv der Mathematik, 58(5):486-491, 1992.

[82] Q.-b. Zheng and C.-z. Cheng. Strong convergence theorem for a family of Lipschitz pseudocontractive mappings in a Hilbert space. Mathematical and Computer Modelling, 48(3-4):480-485, 2008. 
[83] M. Ziegler. Real Computation with Least Discrete Advice: A Complexity Theory of Nonuniform computability. Annals of Pure and Applied Logic, 163(8):1108 - 1139, 2012. Continuity, Computability, Constructivity: From Logic to Algorithms.

This work is licensed under the Creative Commons Attribution-NoDerivs License. To view a copy of this license, visit http://creativecommons.org/licenses/by-nd/2.0/ or send a letter to Creative Commons, 171 Second St, Suite 300, San Francisco, CA 94105, USA, or Eisenacher Strasse 2, 10777 Berlin, Germany 